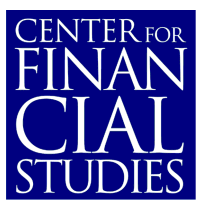

No. $2002 / 10$

\title{
Mixed Normal Conditional Heteroskedasticity
}

\author{
Markus Haas, Stefan Mittnik and
}

Marc S. Paolella

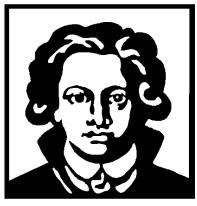

Center for Financial Studies 


\title{
Mixed Normal Conditional Heteroskedasticity
}

\author{
Markus Haas, Stefan Mittnik and Marc S. Paolella*
}

September 2002

\begin{abstract}
:
Both unconditional mixed-normal distributions and GARCH models with fat-tailed conditional distributions have been employed for modeling financial return data. We consider a mixed-normal distribution coupled with a GARCH-type structure which allows for conditional variance in each of the components as well as dynamic feedback between the components. Special cases and relationships with previously proposed specifications are discussed and stationarity conditions are derived. An empirical application to NASDAQindex data indicates the appropriateness of the model class and illustrates that the approach can generate a plausible disaggregation of the conditional variance process, in which the components' volatility dynamics have a clearly distinct behavior that is, for example, compatible with the well-known leverage effect.
\end{abstract}

JEL Classification: C22, C51, G10

Keywords: Finance, GARCH, Kurtosis, Skewness, Stationarity.

* Markus Haas and Marc S. Paolella, Institute of Statistics and Econometrics, University of Kiel, Olshausenstr. 40, 24118 Kiel, Germany

Stefan Mittnik, Institute of Statistics and Econometrics, University of Kiel, Olshausenstr. 40, 24118 Kiel, Germany and Center for Financial Studies, Taunusanlage 6, 60329 Frankfurt am Main, Germany 


\section{Introduction}

Although Generalized Autoregressive Conditional Heteroskedastic Normal (GARCH) models and their numerous extensions can account for a substantial portion of both the volatility clustering and excess kurtosis found in financial return series, a GARCH-type model has yet to be constructed for which the filtered residuals consistently fail to exhibit clear-cut signs of nonnormality. On the contrary, it appears that the vast majority of GARCH-type models, when fit to returns over weekly and shorter horizons, imply quite heavy-tailed conditional innovation distributions. Moreover, there is a growing awareness of skewness in both unconditional and conditional return distributions. ${ }^{1}$ A natural way of accommodating such stylized facts is to specify a GARCH-type structure driven by iid innovations from a fat-tailed and, possibly, asymmetric distribution. A sizeable and growing number of candidate densities exist, a number of which are considered in the application below. Moreover, building on work by Hansen (1994), the studies of Paolella (1999), Harvey and Siddique (1999), Brännäs and Nordman (2001), and Rockinger and Jondeau (2002) employ autoregressive-type structures to allow for time variation in the skewness and, in some cases, also kurtosis. Thus, while not as blatant as volatility clustering and heavy tails, time-varying skewness has emerged as another stylized fact of asset returns.

In this paper, we investigate a model which incorporates the original assumption of normal innovations, yet can still adequately capture all three aforementioned stylized facts. Specifically, we let the conditional distribution be a mixture of normals (in short, MN) and extend the usual GARCH structure by modeling the dynamics in volatility by a system of equations which permits feedback between the mixture components. With one component, the model reduces to the Normal-GARCH model originally proposed in Bollerslev (1986). The excess kurtosis, which plagues Normal-GARCH specifications, can be adequately modeled with only two components. In addition, with more than one component, time-varying skewness is induced, i.e., it is inherent in the model without requiring explicit specification of a conditional skewness process. Moreover, the model can capture the leverage effect. These aspects will be demonstrated in the empirical example below.

The MN formulation also allows for a plausible interpretation of two or more heterogeneous groups of market participants. For example, "bullish" and "bearish" investors could behave differently. Shleifer and Summers (1990) distinguish between "arbitrageurs" or "ratio-

\footnotetext{
${ }^{1}$ See, for example, Kane (1977), Friend and Westerfield (1980), Rozelle and Fielitz (1980), Simkowitz and Beedles (1980), St. Pierre (1993), Mittnik and Rachev (1993), Franses and van Dijk (1996), Peiró (1999), and Harvey and Siddique (1999).
} 
nal speculators" and "noise traders" who react differently to arriving news. Moreover, noise traders may fall into different subgroups, as in Lux (1997), who distinguishes between optimistic and pessimistic naive traders ${ }^{2}$ and shows that the interaction between fundamentalists and chartists may be a source of time-varying second moments. The proposed model class is not only appealing in that it can allow for heterogeneous agents, it also provides, in the empirical application considered, a superior fit compared to competing models. Thus, apart from a viewpoint of financial theory, it will be of interest to practitioners such as risk managers.

The proposed model indeed has some similar characteristics to Markov switching models, which have undoubtedly grown in importance (and complexity) since the seminal work of Hamilton $(1988,1989)$ (see, for example, Hamilton and Susmel, 1994; Gray, 1996; and Dueker, 1997). However, compared to the aforementioned GARCH-type models with fat-tailed innovation distributions, these models have not been shown to be advantageous with respect to either estimation or, more importantly, out-of-sample forecasting (Pagan and Schwert, 1990; Hamilton and Susmel, 1994; Dacco and Satchell, 1999). Nor is the notion of a constant set of unique and recurring regimes any more plausible in a financial context than the decomposition considered herein. An approach similar to ours has recently been explored by Wong and $\mathrm{Li}$ (2001), who also argue against use of a latent Markov structure; see Section 5 below for some discussion of their work.

The remainder of this paper is as follows. Section 2 reviews relevant properties of unconditional MN distributions, presents the MN-GARCH model and discusses various special cases. Section 3 details stationarity conditions. An empirical application is presented in Section 4. Section 5 provides concluding remarks.

\section{$2 \quad$ Mixed Normal Models}

The MN distribution has a long and illustrious history in statistics. Its use for modeling heavytailed distributions apparently dates back to 1886, when the mathematician, astronomer and economist Simon Newcomb used it in his astronomical studies (Newcomb, 1980). After the seminal work of Pearson in 1894 on the moments estimator for the univariate normal mixture with two components, maximum likelihood (ML) estimation has become very popular with the advent of the EM algorithm of Dempster et al. (1977), while exact Bayesian analysis of mixtures has become feasible after the introduction of the Gibbs sampler of Geman and Geman (1984) into the statistical mainstream by Gelfand and Smith (1990) and Gelfand et al.

\footnotetext{
${ }^{2}$ In Lux (1997), noise traders also differ with respect to their trading-strategies: Some try to find out "the mood of the market", others follow various "technical" trading rules.
} 
(1990). Further historical aspects, modern inferential methods, and discussion of applications associated with mixtures of normals are given in Titterington et al. (1985), McLachlan and Basford (1988), and McLachlan and Peel (2000).

\subsection{Unconditional Mixed Normal Distribution}

A random variable $Y$ is said to have a univariate (finite) normal mixture distribution if its unconditional density is given by

$$
f(y)=\sum_{j=1}^{k} \lambda_{j} \phi\left(y ; \mu_{j}, \sigma_{j}^{2}\right),
$$

where $\lambda_{j}>0, j=1, \ldots, k, \sum_{j=1}^{k} \lambda_{j}=1$, are the mixing weights and

$$
\phi\left(y ; \mu_{j}, \sigma_{j}^{2}\right)=\frac{1}{\sqrt{2 \pi} \sigma_{j}} \exp \left\{-\frac{1}{2}\left(\frac{y-\mu_{j}}{\sigma_{j}}\right)^{2}\right\}, \quad j=1, \ldots, k,
$$

are the component densities. The normal mixture has finite moments of all orders, with expected value and variance given by

$$
\mu=\mathrm{E}(Y)=\sum_{j=1}^{k} \lambda_{j} \mu_{j}, \quad m_{2}=\operatorname{Var}(Y)=\sum_{j=1}^{k} \lambda_{j} \sigma_{j}^{2}+\sum_{j=1}^{k} \lambda_{j}\left(\mu_{j}-\mathrm{E}(Y)\right)^{2} .
$$

Owing to its great flexibility (see, for example, the various density plots in Marron and Wand, 1992), the MN has also been found useful for describing the unconditional distribution of asset returns (cf. Fama, 1965; Kon, 1984; Akgiray and Booth, 1987; and Tucker and Pond, 1988). Indeed, even a two-component mixture is rather capable of exhibiting the skewness and kurtosis typical of financial data. To demonstrate the skewness property, let $Y$ be a $k^{-}$ component mixed normal random variable with mean $\mu=\sum_{j=1}^{k} \lambda_{j} \mu_{j}$. Since for constant $a$, $\int(y-a)^{n} \phi\left(y ; \mu, \sigma^{2}\right) d y=\int y^{n} \phi\left(y ; \mu-a, \sigma^{2}\right) d y$, we have

$$
\begin{aligned}
m_{3}=\mathrm{E}\left[(Y-\mu)^{3}\right] & =\sum_{j=1}^{k} \lambda_{j}\left(\mu_{j}-\mu\right)^{3}+3 \sum_{j=1}^{k} \lambda_{j} \sigma_{j}^{2}\left(\mu_{j}-\mu\right) \\
& =\sum_{j=1}^{k} \lambda_{j}\left(\mu_{j}-\mu\right)^{3}+3 \sum_{j=1}^{k} \sum_{i<j} \lambda_{j} \lambda_{i}\left(\sigma_{j}^{2}-\sigma_{i}^{2}\right)\left(\mu_{j}-\mu_{i}\right),
\end{aligned}
$$

which shows that common component means, i.e., $\mu_{1}=\cdots=\mu_{k}=\mu$, imply symmetry. For $k=2$, the above expression becomes

$$
\begin{aligned}
m_{3} & =\frac{\lambda_{1}}{\lambda_{2}}\left(1-\frac{\lambda_{1}}{\lambda_{2}}\right)\left(\mu_{1}-\mu\right)^{3}+3 \lambda_{1}\left(\mu_{1}-\mu\right)\left(\sigma_{1}^{2}-\sigma_{2}^{2}\right) \\
& =\lambda_{1} \lambda_{2}\left[\left(\lambda_{2}-\lambda_{1}\right)\left(\mu_{1}-\mu_{2}\right)^{3}+3\left(\mu_{1}-\mu_{2}\right)\left(\sigma_{1}^{2}-\sigma_{2}^{2}\right)\right] .
\end{aligned}
$$


If $\mu_{1} \neq \mu_{2}$, then it is necessary and sufficient that $\lambda_{1} \neq \lambda_{2}$ and/or $\sigma_{1}^{2} \neq \sigma_{2}^{2}$ for $Y$ to be asymmetric. ${ }^{3}$

With regard to kurtosis, let $Y$ be a $k$-component mixed normal random variable but with $\mu_{1}=\ldots=\mu_{k}=\mu$, so that $\mathrm{E}(Y)=\sum_{j} \lambda_{j} \mu_{j}=\mu$. Then, from Jensen's inequality, $\sum_{j} \lambda_{j} \sigma_{j}^{4}=$ $\sum_{j} \lambda_{j}\left(\sigma_{j}^{2}\right)^{2}>\left(\sum_{j} \lambda_{j} \sigma_{j}^{2}\right)^{2}$, so that ${ }^{4}$

$$
\gamma_{2}=\frac{m_{4}}{m_{2}^{2}}=\frac{\mathrm{E}\left[(Y-\mu)^{4}\right]}{\left[\mathrm{E}\left[(Y-\mu)^{2}\right]\right]^{2}}=3 \frac{\sum_{j} \lambda_{j} \sigma_{j}^{4}}{\left(\sum_{j} \lambda_{j} \sigma_{j}^{2}\right)^{2}}>3 .
$$

An advantage of the MN model not shared by other distributional assumptions is that it lends itself to economic interpretation in several ways. A mixture of two or more normals could arise from different groups of actors, with one group acting, for example, more volatile than the other or, possibly, processing market information differently. Considering unconditional distributions, Kon (1984), for example, argues that returns on individual stocks may be drawn from a noninformation distribution, a firm-specific distribution and a market-wide information distribution, i.e., a three component mixture.

The MN model can also be appropriate for samples where the components follow a repeating sequence in generating observations. As an example, day-of-the-week effects, as mentioned by Fama (1965), are a possible source of mixture distributions. More specifically, political and economic news arrivals occur continuously, and, if they are assimilated continuously by investors, the variance of the distribution of price changes between two points in time would be proportional to the actual number of days elapsed (as in the Monday-effect). By analyzing corresponding subsamples, however, Fama (1965) found that the Monday-effect does not give rise to the observed departure from normality. However, the mixture may still be interpreted as representing trading days of different types: A component with relatively low variance, for example, could represent "business as usual" - typically associated with a large mixing weight - while components with high variances and smaller weights could correspond to times of high volatility caused by the arrival of substantive new information.

\footnotetext{
${ }^{3}$ Necessity is rather obvious. Sufficiency follows from the fact that symmetry implies $m_{3}=0$. If $\mu_{1}=$ $\mu \pm \lambda_{2} \sqrt{3\left(\sigma_{1}^{2}-\sigma_{2}^{2}\right) /\left(\lambda_{1}-\lambda_{2}\right)}=: \mu \pm \lambda_{2} \tau$, then $m_{3}=0$ but the density is not symmetric. Symmetry, i.e., $f(\mu+y)=f(\mu-y)$, would imply that, for all $y$,

$$
\lambda_{1} \phi\left(y ; \pm \lambda_{2} \tau, \sigma_{1}^{2}\right)+\lambda_{2} \phi\left(y ; \mp \lambda_{1} \tau, \sigma_{2}^{2}\right)=\lambda_{1} \phi\left(y ; \mp \lambda_{2} \tau, \sigma_{1}^{2}\right)+\lambda_{2} \phi\left(y ; \pm \lambda_{1} \tau, \sigma_{2}^{2}\right),
$$

which does not hold for any $\tau \neq 0$ because the class of finite normal mixtures is identifiable (Teicher, 1963). That the density can only be symmetric about its mean is clear; see, e.g., Dudewicz and Mishra (1988, pp. 216-217).

${ }^{4}$ If, however, the means are far enough apart (so that the density is not highly peaked around its center), the kurtosis can actually be less than three.
} 


\subsection{Conditionally Heteroskedastic MN Processes}

Time series $\left\{\epsilon_{t}\right\}$ is generated by a $k$-component Mixed Normal $\operatorname{GARCH}(p, q)$ process, or, in short, MN-GARCH, if the conditional distribution of $\epsilon_{t}$ is a $k$ component MN with zero mean, i.e.,

$$
\epsilon_{t} \mid \Psi_{t-1} \sim \mathrm{MN}\left(\lambda_{1}, \ldots, \lambda_{k}, \mu_{1}, \ldots, \mu_{k}, \sigma_{1 t}^{2}, \ldots, \sigma_{k t}^{2}\right),
$$

where $\Psi_{t}$ is the information set at time $t ; \lambda_{i} \in(0,1), i=1, \ldots, k, \sum_{i=1}^{k} \lambda_{i}=1 ;$ and $\mu_{k}=$ $-\sum_{i=1}^{k-1}\left(\lambda_{i} / \lambda_{k}\right) \mu_{i}$. Furthermore, the $k \times 1$ vector of component variances, denoted by $\sigma_{t}^{(2)}$, evolves according to

$$
\sigma_{t}^{(2)}=\alpha_{0}+\sum_{i=1}^{q} \alpha_{i} \epsilon_{t-1}^{2}+\sum_{j=1}^{p} \beta_{j} \sigma_{t-j}^{(2)},
$$

where $\sigma_{t}^{(2)}=\left[\sigma_{1 t}^{2}, \sigma_{2 t}^{2}, \ldots, \sigma_{k t}^{2}\right]^{T} ; \alpha_{i}=\left[\alpha_{i 1}, \alpha_{i 2}, \ldots, \alpha_{i k}\right]^{T}, i=0, \ldots, q ;$ and $\beta_{j}, j=1, \ldots, p$, are $k \times k$ matrices with typical element $\beta_{j, m n}$. Restrictions $\alpha_{0}>0, \alpha_{i} \geq 0, i=0, \ldots, q$, and $\beta_{j} \geq 0, j=1, \ldots, p$, are assumed. ${ }^{5}$ They correspond to the non-negativity conditions of Bollerslev (1986) for the Normal-GARCH model, although they may be unnecessarily strong (Nelson and Cao, 1992). They are, however, necessary for the diagonal MN-GARCH(1,1) model, a useful special case introduced and employed below.

Using lag-operator notation, $L^{q} y_{t}=y_{t-q}$, an MN-GARCH process can be written as

$$
\left(I_{k}-\beta(L)\right) \sigma_{t}^{(2)}=\alpha_{0}+\alpha(L) \epsilon_{t}^{2},
$$

where $\beta(L)=\sum_{j=1}^{p} \beta_{j} L^{j} ; \alpha(L)=\sum_{i=1}^{q} \alpha_{i} L^{i} ;$ and $I_{k}$ is the identity matrix of dimension $k$.

As is common, a mean equation can also be introduced to incorporate exogenous variables and/or lagged values via an $\operatorname{ARMA}(u, v)$ structure. In particular, an ARMA-MN-GARCH model for variable $r_{t}$ refers to a process with mean equation

$$
r_{t}=a_{0}+\sum_{i=1}^{u} a_{i} r_{t-i}+\epsilon_{t}+\sum_{j=1}^{v} b_{j} \epsilon_{t-j}
$$

with constant $a_{0}$, AR parameters $a_{1}, \ldots, a_{u}$, MA parameters $b_{1}, \ldots, b_{v}$, and with $\epsilon_{t} \mid \Psi_{t-1}$ given by (4) and (6).

\subsection{Special Cases}

\subsubsection{Diagonal MN-GARCH}

A particularly interesting special case for modeling asset returns arises by restricting matrix $\beta(L)$ in (6) to be diagonal (subsequently referred to as a diagonal MN-GARCH process). In

\footnotetext{
${ }^{5}$ In case of vectors and matrices, symbol $\geq$ indicates elementwise inequality.
} 
addition to allowing for a clear interpretation of the dynamics of the component variances, we find - not only for the example reported below - that it tends to be preferred over the full model when employing various model-selection criteria.

\subsubsection{Partial MN-GARCH}

With the interpretation of different groups of actors in mind, it is conceivable that the market is driven by a mixture in which some components exhibit constant variance. Such components could be associated with informed traders, whereas the dynamic components could be due to noise traders, possibly overreacting to news. Below we consider diagonal partial models, where a model denoted by $\mathrm{MN}(k, g), g \leq k$, uses $k$ component densities, $g$ of which follow a GARCH process and $k-g$ components are restricted to be constant. If, for example, models with $g=1$ fit the data well, then the unconditional properties of the normal mixture (skewness and kurtosis) account for most of the improvement relative to the standard GARCH model with conditional normality, and volatility clustering is adequately captured by introducing one GARCH component.

\subsubsection{Symmetric $\mathrm{MN}_{s}-\mathrm{GARCH}$}

We also entertain models for which all the component means are restricted to be zero, i.e., $\mu_{1}=\mu_{2}=\cdots \mu_{k}=0$, which imposes a symmetric conditional error distribution. These are denoted by $\mathrm{MN}_{s}(k, g)-\mathrm{GARCH}$. Because both the conditional innovations and the GARCH structure are symmetric, the unconditional error distribution will also be symmetric.

\subsection{Relationship with Other MN-GARCH Specifications}

To the best of our knowledge, Vlaar and Palm (1993) and Palm and Vlaar (1997) first suggested the normal mixture in a GARCH context. The model they proposed is restricted such that, for all $t, \sigma_{2 t}^{2}=\sigma_{1 t}^{2}+\delta^{2}$ (cf. the parameterization in Ball and Torous, 1983) ${ }^{6}$ and can be nested in (5). In our notation, it takes the form

$$
\left[\begin{array}{c}
\sigma_{1 t}^{2} \\
\sigma_{2 t}^{2}
\end{array}\right]=\left[\begin{array}{c}
\alpha_{01} \\
\alpha_{01}+\delta^{2}
\end{array}\right]+\left[\begin{array}{c}
\alpha_{11} \\
\alpha_{11}
\end{array}\right] \epsilon_{t-1}^{2}+\left[\begin{array}{cc}
\beta_{11} & 0 \\
\beta_{11} & 0
\end{array}\right]\left[\begin{array}{c}
\sigma_{1, t-1}^{2} \\
\sigma_{2, t-1}^{2}
\end{array}\right],
$$

which permits skewness by allowing the component means to differ from zero.

\footnotetext{
${ }^{6}$ Vlaar and Palm (1993, p. 357) motivate this specification by arguing that "...this procedure is preferred to that of independent variances, since it seems reasonable to assume that the same GARCH effect is present in all variances."
} 
Bauwens et al. (1999a) consider a MN-GARCH model with two components, in which the component variances are proportional to each other, i.e., for all $t, \sigma_{2 t}^{2}=\tau \sigma_{1 t}^{2}$, specializing (5) to $^{7}$

$$
\left[\begin{array}{c}
\sigma_{1 t}^{2} \\
\sigma_{2 t}^{2}
\end{array}\right]=\left[\begin{array}{c}
\alpha_{0} \\
\tau \alpha_{0}
\end{array}\right]+\left[\begin{array}{c}
\alpha_{1} \\
\tau \alpha_{1}
\end{array}\right] \epsilon_{t-1}^{2}+\left[\begin{array}{cc}
\beta_{11} & 0 \\
\tau \beta_{11} & 0
\end{array}\right]\left[\begin{array}{c}
\sigma_{1, t-1}^{2} \\
\sigma_{2, t-1}^{2}
\end{array}\right] .
$$

It may be argued that the proportionality property is less appealing, since it implies that both components exhibit essentially the same dynamic behavior and does not allow for two (or more) differently acting groups of market participants having, for example, different speeds of adjustment. This feature also applies to the Palm and Vlaar specification.

Another special MN-GARCH model has been proposed in Lin and Yeh (2000). Their model is also characterized by imposing the same dynamics on each component variance, i.e., only the constants $\alpha_{0 j}, j=1, \ldots, k$, in the GARCH equations are component-specific, while the coefficients of lagged squared error terms and variances are the same in each equation. For $k=2$, this amounts to restricting (5) to

$$
\left[\begin{array}{l}
\sigma_{1 t}^{2} \\
\sigma_{2 t}^{2}
\end{array}\right]=\left[\begin{array}{l}
\alpha_{01} \\
\alpha_{02}
\end{array}\right]+\left[\begin{array}{l}
\alpha_{11} \\
\alpha_{11}
\end{array}\right] \epsilon_{t-1}^{2}+\left[\begin{array}{cc}
\beta_{11} & 0 \\
0 & \beta_{11}
\end{array}\right]\left[\begin{array}{l}
\sigma_{1, t-1}^{2} \\
\sigma_{2, t-1}^{2}
\end{array}\right] .
$$

Finally, it should be noted that MN-GARCH processes are related to the $t$-GARCH model (Bollerslev, 1987) in that the $t$ distribution can be represented as an infinite gamma-mixture of normals.

\section{Stationarity and Persistence}

\subsection{Weak Stationarity}

\subsubsection{The General Case}

Given the existence of the unconditional expectation $\mathrm{E} \sigma_{t}^{(2)}$, standard calculations using the law of iterated expectations show that

$$
\mathrm{E} \sigma_{t}^{(2)}=\left[I-\beta(1)-\alpha(1) \lambda^{T}\right]^{-1}\left[\alpha_{0}+\alpha(1) c\right]
$$

where (see Appendix A for derivation)

$$
c=\sum_{j=1}^{k} \lambda_{j} \mu_{j}^{2}=\frac{1}{\lambda_{k}}\left[\sum_{j=1}^{k-1} \lambda_{j} \mu_{j}^{2}-\sum_{j<r<k} \sum_{j} \lambda_{r}\left(\mu_{j}-\mu_{r}\right)^{2}\right] .
$$

\footnotetext{
${ }^{7}$ Alternatively, because $\sigma_{2, t-1}^{2}=\tau \sigma_{1, t-1}^{2}$, we could define $\beta=\beta_{11} I_{2}$.
} 
As relationship (8) suggests and Appendix B shows, the necessary and sufficient condition for the existence of the unconditional variance is

$$
\operatorname{det}\left[I-\beta(1)-\alpha(1) \lambda^{T}\right]>0 \text {. }
$$

An interpretation of (9) is provided in Appendix B. Condition (9) assumes a simple form in the special diagonal MN-GARCH case, which is discussed next.

\subsubsection{The Diagonal Case}

For diagonal MN-GARCH processes, defining $\tilde{\beta}_{j}=1-\sum_{i=1}^{p} \beta_{i, j j}$, we have, from (B.7), that

$$
\begin{aligned}
\operatorname{det}\left[I-\beta(1)-\alpha(1) \lambda^{T}\right] & =\operatorname{det}[I-\beta(1)]-\sum_{j=1}^{k} \lambda_{j} \operatorname{det} B(1)_{j} \\
& =\left[1-\sum_{j=1}^{k} \frac{\lambda_{j}}{\tilde{\beta}_{j}} \sum_{i=1}^{q} \alpha_{i j}\right] \prod_{j=1}^{k} \tilde{\beta}_{j} \\
& =\left[\sum_{j=1}^{k} \frac{\lambda_{j}}{\tilde{\beta}_{j}}\left(1-\sum_{i=1}^{q} \alpha_{i j}-\sum_{i=1}^{p} \beta_{i, j j}\right)\right] \prod_{j=1}^{k} \tilde{\beta}_{j},
\end{aligned}
$$

where matrix $B(1)_{j}$ is defined in (B.2). This last expression implies that it is not necessary that the inequalities $1-\sum_{i=1}^{q} \alpha_{i j}-\sum_{i=1}^{p} \beta_{i, j j}>0$ have to hold for all $j \in\{1, \ldots, k\}$, but rather for their weighted sum with the $j^{\text {th }}$ weight being given by $\lambda_{j} / \tilde{\beta}_{j}$ and the weights not summing to one. ${ }^{8}$ The mixing weight of each component is inflated by the component's contribution to the deterministic part of $\sigma_{t}^{(2)}$ in (5). This condition is stronger than just $\sum_{j=1}^{k} \lambda_{j}\left(1-\sum_{i=1}^{q} \alpha_{i j}-\sum_{i=1}^{p} \beta_{i, j j}\right)>0$ due to the feedback between the components.

By writing requirement (10) as

$$
1-\sum_{j=1}^{k} \lambda_{j} \sum_{i=1}^{q} \alpha_{i j}\left(1-\sum_{i=1}^{p} \beta_{i, j j}\right)^{-1}>0,
$$

we see that it is a direct generalization of the well-known stationarity condition stated in Bollerslev (1986), which can be expressed as

$$
1-\sum_{i=1}^{q} \alpha_{i}\left(1-\sum_{i=1}^{p} \beta_{i}\right)^{-1}>0 .
$$

Using (B.5), the unconditional variance of a diagonal MN-GARCH process becomes

$$
\begin{aligned}
\mathrm{E}\left(\epsilon_{t}^{2}\right) & =\lambda^{T} \mathrm{E}\left(\sigma_{t}^{(2)}\right)+c=c \frac{\operatorname{det}\left[I-\beta+\alpha_{0} \lambda^{T} / c\right]}{\operatorname{det}\left[I-\beta-\alpha_{1} \lambda^{T}\right]} \\
& =\frac{c+\sum_{j} \lambda_{j} \alpha_{0 j} / \tilde{\beta}_{j}}{1-\sum_{j} \lambda_{j} / \tilde{\beta}_{j} \sum_{i} \alpha_{i j}}=\frac{c+\sum_{j} \lambda_{j} \alpha_{0 j} / \tilde{\beta}_{j}}{\sum_{j} \lambda_{j} / \tilde{\beta}_{j}\left(1-\sum_{i} \alpha_{i j}-\sum_{i} \beta_{i, j j}\right)} .
\end{aligned}
$$

\footnotetext{
${ }^{8}$ Clearly, $\prod_{j} \tilde{\beta}_{j}>0$ must be assumed, since otherwise the deterministic part of difference equation (5) would be explosive.
} 
For $k=1$, this reduces to $\mathrm{E}\left(\epsilon_{t}^{2}\right)=\alpha_{0} /\left(1-\sum_{i} \alpha_{i}-\sum_{i} \beta_{i}\right)$, as in Bollerslev (1986).

According to (10), the process can have finite variance even though some components are not covariance stationary, as long as the corresponding weights are sufficiently small. This result is similar to the condition for strict stationarity given by Francq et al. (2001) for a regime-switching GARCH $(1,1)$ model. They show that, in this model, the condition derived by Nelson (1991) for the single-regime GARCH model need not hold in each regime but for a weighted average of the $\mathrm{GARCH}$-parameters in each regime, where the weights are the stationary probabilities of the Markov chain.

\subsection{Measuring Volatility Persistence}

As is demonstrated in Appendix $\mathrm{B}$, the largest eigenvalue, $\rho_{\max }$, of matrix

$$
\Phi=\left[\begin{array}{ccccc}
\beta_{1}+\alpha_{1} \lambda^{T} & \beta_{2}+\alpha_{2} \lambda^{T} & \cdots & \beta_{r-1}+\alpha_{r-1} \lambda^{T} & \beta_{r}+\alpha_{r} \lambda^{T} \\
I_{k} & 0_{k} & \cdots & 0_{k} & 0_{k} \\
0_{k} & I_{k} & & 0_{k} & 0_{k} \\
\vdots & \vdots & \ddots & \vdots & \vdots \\
0_{k} & 0_{k} & \cdots & I_{k} & 0_{k}
\end{array}\right],
$$

with $r=\max \{p, q\}$ and $0_{k}$ denotes a $k \times k$ matrix of zeros, can serve as a measure of volatility persistence, since the impact of past variances declines geometrically at the rate $\rho_{\max }$. In the case of an MN-GARCH$(1,1)$ model, this is the largest eigenvalue of the matrix $\beta(1)+\alpha(1) \lambda^{T}$. Analogous to the expression for the single component case, i.e., a Normal-GARCH $(1,1)$ model (Bollerslev et al., 1994), the conditional expectation of future variances in this model is given by

$$
\mathrm{E}\left[\sigma_{t+k}^{(2)} \mid \Psi_{t-1}\right]=\sigma^{(2)}+\left(\beta(1)+\alpha(1) \lambda^{T}\right)^{k}\left(\sigma_{t}^{(2)}-\sigma^{(2)}\right),
$$

where, from (8),

$$
\sigma^{(2)}=\mathrm{E}\left(\sigma_{t}^{(2)}\right)=\left[I-\beta(1)-\alpha(1) \lambda^{T}\right]^{-1}\left(\alpha_{0}+\alpha_{1} c\right)
$$

and $\left[\beta(1)+\alpha(1) \lambda^{T}\right]^{k}$ tends to zero geometrically with rate $\rho_{\max }$.

\section{Conditional Heteroskedasticity of NASDAQ Returns}

We investigate the daily returns on the NASDAQ index from its inception in February 1971 to June 2001, a sample of $T=7,681$ observations. ${ }^{9}$ Continuously compounded percentage

\footnotetext{
${ }^{9}$ The data were obtained from the internet site http://www.marketdata.nasdaq.com, maintained by the Economic Research Department of The National Association of Securities Dealers, Inc.
} 


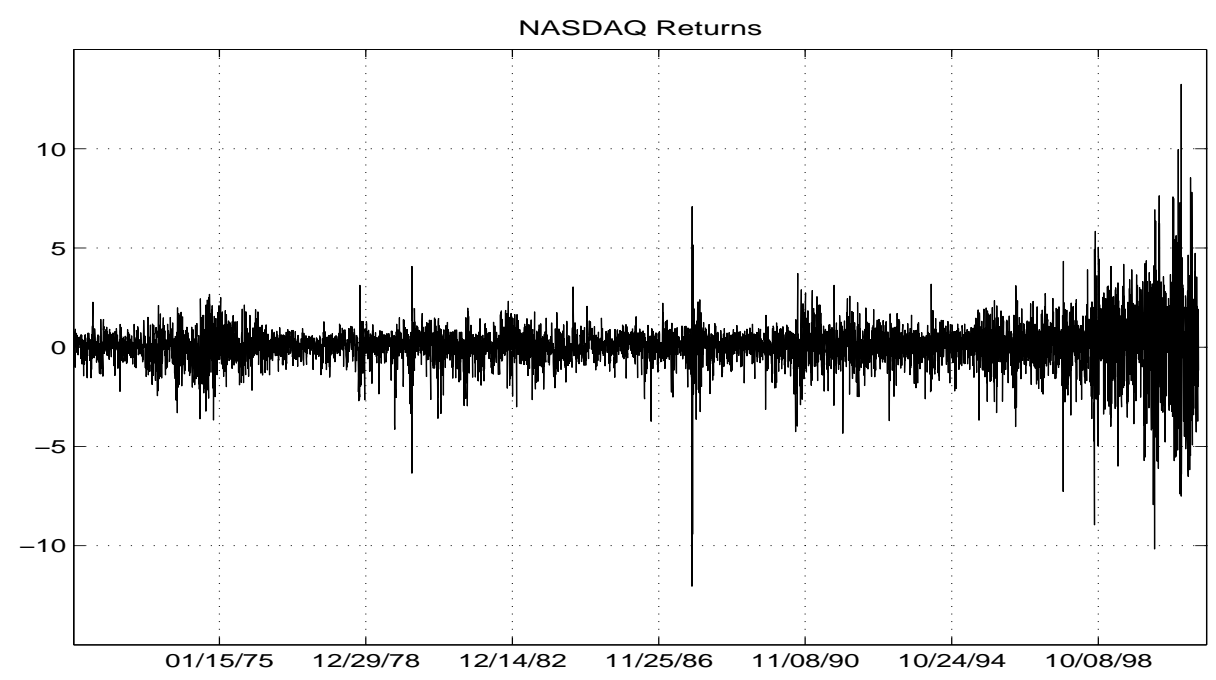

Figure 1: Percentage Returns on NASDAQ Index.

returns, $r_{t}=100\left(\log P_{t}-\log P_{t-1}\right)$, are considered, where $P_{t}$ denotes the index level at time $t$. Figure 1 shows a plot of the return series. While the usual stylized fact of strong volatility clustering is apparent from Figure 1, it is not as obvious that the data are also negatively skewed. The usual measure for asymmetry involving the third moment of the data (let alone its asymptotically valid standard error under normality) is virtually meaningless to report, given that 3rd and higher moments of financial data may not exist. In this case, estimating an unconditional Student's $t$ distribution resulted in 2.4 degrees of freedom (and approximate standard error 0.08). One possible way to infer if asymmetry is statistically significant is to use a flexible parametric density which allows for asymmetry and fit it both restricted and unrestricted, from which a likelihood ratio test for asymmetry can be constructed. This was done using the noncentral $t$ distribution, as suggested for use in a financial modeling context by Harvey and Siddique (1999); the asymmetric generalized $t$ distribution in Mittnik and Paolella (2000); and the stable Paretian distribution (see, e.g., Mittnik, Paolella and Rachev, 2000). The likelihood ratio values were 69, 73 and 66, respectively, which are clearly tremendously significant at any conventional testing level.

Sample autocorrelation plots of Normal-GARCH(1,1) residuals (not shown) suggest a low order AR model for the mean equation. The Bayesian Information Criterion (BIC) favors an $\operatorname{AR}(3)$, which will accompany all GARCH structures estimated below. ${ }^{10}$

\footnotetext{
${ }^{10} \operatorname{All} \operatorname{ARMA}(r, s)-\operatorname{GARCH}(1,1)$ models for combinations $r+s \leq 4$ were estimated, assuming conditionally normal innovations.
} 


\subsection{Estimation Issues}

We estimate the ARMA-MN-GARCH model by conditional ML, conditioning, due to the ARMA structure (7), on the first $u$ return observations and set the first $v$ values of $\epsilon_{t}$ to zero and, for the GARCH structure, set the initial values of $\sigma_{t}^{(2)}$ and $\epsilon_{t}^{2}$ equal to their unconditional expectations given in (8). ${ }^{11}$ Because it is not clear what the "typical" parameter values would be for the GARCH structure with $k \geq 2$ components when applied to financial return series, we simply set the starting values to $\lambda_{i}=1 / k, \alpha_{01}=\ldots=\alpha_{0 k}=0.05, \alpha_{11}=\ldots=\alpha_{1 k}=0.1$, $\beta_{1,11}=\ldots=\beta_{1, k k}=0.8$ and the off-diagonal elements of $\beta$ matrices to zero. For several real data sets including the one used below (as well as many simulated series), these proved adequate, with convergence occurring usually within 20 to 50 iterations. Use of other, even very unrealistic, starting values led in virtually all cases to the same estimates.

Bayesian inference via Markov Chain Monte Carlo methods such as the Metropolis Hastings algorithm (see Chib and Greenberg, 1996, and Bauwens et al., 1999b, and the references therein) is theoretically possible, but for the large sample sizes typically available in financial applications and the lack of strong prior information, conditional ML estimation should yield very similar results. Furthermore, obtaining the ML estimates is computationally easier, both in terms of programming effort as well as in run time and assessment of convergence. For the diagonal model discussed below, an EM algorithm could also be constructed, but would offer little, if any gain, given the slow convergence of the method, and because each M-step would itself require numerical optimization.

\subsection{Determining the Number of Mixture Components}

For mixture models in general, the number of required component densities is unknown and needs to be empirically determined. Unfortunately, standard test theory breaks down in this context; see, for example, Wolfe (1971), Everitt and Hand (1981), Aitkin et al. (1981), Hartigan (1985), Ghosh and Sen (1985) McLachlan and Peel (2000, Ch. 6), and Chen et al. (2001). These authors perform and refer to simulation studies suggesting that the asymptotic distribution of the usual likelihood ratio test statistic mimics a $\chi^{2}$ distribution with degrees of freedom larger than the number of fixed parameters under the null. This draws into question the results of Kon (1984), in which standard theory is used to provide evidence that some stocks are best modeled by a mixture of four components. Similar criticism applies to Kim and Kon (1994), although the values of the likelihood ratio statistics reported there (ranging

\footnotetext{
${ }^{11}$ The quasi-Newton maximization method available in Matlab (version 5.2, function fminu) was used, with (automatically computed) numeric gradient and Hessian, and a convergence criterion of 0.0001.
} 
from 423 to 1854) are high enough to keep their conclusions valid under more appropriate methods of model selection.

Standard model selection criteria such as the AIC (Akaike, 1973) and the BIC (Schwarz, 1978) are widely used in the GARCH literature and can be used to compare models with differing numbers of components. For a model with $K$ parameters and log-likelihood, $L$, evaluated at the maximum likelihood estimator, $\mathrm{AIC}=-2 L+2 K$ and $\mathrm{BIC}=-2 L+K \log T$, with BIC being more conservative than AIC in that it favors more parsimonious models. Because these measures rely on the same conditions employed in the asymptotic theory of the likelihood ratio test, their small and large sample properties are likewise not known. However, the literature on mixtures provides some encouraging evidence in the context of unconditional models, suggesting that the BIC provides a reasonably good indication for the number of components (see, in particular, Dasgupta and Raftery, 1998; Fraley and Raftery, 1998; Leroux, 1992; Roeder and Wassermann, 1997; and McLachlan and Peel, 2000, Ch. 6). According to Kass and Raftery (1995), a BIC difference of less than two corresponds to "not worth more than a bare mention", while differences between two and six imply positive evidence, differences between six and ten give rise to strong evidence, and differences greater than ten invoke very strong evidence. The results of Mittnik and Paolella (2000) suggest that, with respect to out-of-sample prediction, these measures are indeed useful for choosing among GARCH-type models with competing distributional assumptions.

\subsection{Goodness of Fit and Diagnostic Checking}

In addition to the likelihood-based model selection via AIC and BIC, we examine the distributional properties of the residuals of the models. With the MN-GARCH model, it is not possible to directly evaluate the distributional properties of the estimated residuals $\hat{\epsilon}_{t}$ because, even if the model were correctly specified, standardized residuals would not be identically distributed. To circumvent this, we transform the residuals by computing the corresponding value of the conditional cdf, that is,

$$
\hat{u}_{t}=\hat{F}\left(\hat{\epsilon}_{t} \mid \Psi_{t-1}\right), \quad t=1, \ldots, T
$$

Under a correct specification, the transformed residuals, $\hat{u}_{t}$, are iid uniform (Rosenblatt, 1952; see also Diebold et al., 1998). Thus, an inspection of the quantile fit can be based on the $T$ $\hat{u}_{t}$-values. Below, we report for selected $\xi$-values the percentage of $\hat{u}_{t}$-values, denoted by $U_{\xi}$, for which $\hat{u}_{t} \leq \xi$; i.e.,

$$
U_{\xi}=100 \times T^{-1} \sum_{t=1}^{T} \mathcal{I}_{[0, \xi]}\left(\hat{u}_{t}\right),
$$


where $\mathcal{I}$ denotes the indicator function. For a correctly specified model, we expect $U_{\xi} \approx 100 \times \xi$. Also, a histogram of the $\hat{u}_{t}$ 's provides a valuable visual check of the goodness of fit. To formally test for uniformity of the transformed values (12), we use the Pearson goodness-of-fit test, as was suggested by Palm and Vlaar (1997). The test statistic is given by

$$
X^{2}=\sum_{i=1}^{g} \frac{\left(n_{i}-n_{i}^{*}\right)^{2}}{n_{i}^{*}},
$$

where $g$ is the number of (equally spaced) subintervals over the $[0,1]$-interval; $n_{i}$ is the number of observations in interval $i$; and $n_{i}^{*}$ is the expected number of observations under the null hypothesis of uniformity. Below, we will report the results for $g=100$.

If (14) is used to test a simple hypothesis, the statistic has an asymptotic $\chi^{2}$ distribution with $g-1$ degrees of freedom under the null. However, if the hypothesis is composite, the $X^{2}$-values tend to be smaller when evaluated at the estimated rather than the true parameter values. As a consequence, the asymptotic distribution of (14) is actually unknown, but is bounded between the $\chi^{2}(g-K-1)$ and $\chi^{2}(g-1)$ distributions, where $K$ is the number of estimated parameters $^{12}$ (see Stuart et al., 1999, Ch. 25). To reflect the uncertainty about the true asymptotic distribution of $X^{2}$, we will act as if it were $\chi^{2}(g-K-1)$-distributed, so that the test tends to favor models with less parameters resulting in similar fit.

A drawback of the above test is the degree of arbitrariness that is inherent in the choice of the number of classes, $g .{ }^{13}$ In addition, one may wish to test whether the specified distribution captures some specific characteristics of the data such as (conditional) skewness and kurtosis. ${ }^{14}$ This can be accomplished by the further transformation

$$
z_{t}=\Phi^{-1}\left(\hat{u}_{t}\right)
$$

where $\Phi$ is the standard normal cdf, such that the $z_{t}$ 's are iid $\mathrm{N}(0,1)$ distributed, if the underlying model is correct. Berkowitz (2001) shows that inaccuracies in the specified density will be preserved in the transformed data. ${ }^{15}$ Thus, this transformation allows the use of normal probability plots or moment-based normality tests for checking features such as correct specification of skewness and kurtosis.

\footnotetext{
${ }^{12}$ If the parameters are determined by minimizing (14), the exact asymptotic distribution is $\chi^{2}(g-K-1)$.

${ }^{13}$ For example, the use of values between $g=50$ and $g=150$ gave rise to $p$-values below 0.01 in $1 \%, 2 \%, 1 \%$, and $5 \%$ of the cases for models $\mathrm{MN}(2,2), \mathrm{MN}(3,2), \mathrm{MN}(3,3)$, and $\mathrm{MN}(4,4)$, respectively (for the model-notation, see Section 2.3).

${ }^{14}$ As skewness and kurtosis of a mixture model are (complicated) functions of the model parameters, timevariability of the component variances implies time-varying skewness and kurtosis.

${ }^{15}$ Use of values (15) was also advocated by Palm and Vlaar (1997).
} 


\subsection{Competing Models}

In the following comparison, all models entertained share a common $\operatorname{AR}(3)-\operatorname{GARCH}(1,1)$ specification, i.e., following the notation in Section 2.2, $u=3, v=0$ and $p=q=1$. Within the MN-GARCH model class, for a given number of components, $k$, it turns out that the diagonal model was always preferred over the full model when using the BIC criterion. With respect to the AIC, only for $k=2$ was the full model preferred. For this reason, we restrict our attention to the diagonal models in the following analysis. We briefly discuss the characteristics of the full model for $k=2$ and $k=3$ at the end of this section.

In addition to several MN-GARCH specifications, we also fit the $\operatorname{AR}(3)-\operatorname{GARCH}(1,1)$ model assuming a variety of conditional innovation distributions. To save space, we do not reproduce the density specifications here and refer the reader to the corresponding citations provided. Along with the Student's $t$ (Bollerslev, 1987), two asymmetric generalizations are used, namely the non-central $t$ distribution (Harvey and Siddique, 1999) and the so-called $t_{3}$ distribution used in Mittnik and Paolella (2000). Further candidates include the hyperbolic (Eberlein and Keller, 1995; Küchler et al., 1999; Paolella, 1999), the generalized logistic (or EGB2) distribution (Paolella, 1997, Wang et al., 2001) and the asymmetric two-sided Weibull (Mittnik et al., 1998), abbreviated ADW.

Table 1 reports the likelihood-based goodness-of-fit measures for the fitted models and the rankings of the models with respect to each of the criteria. Not surprisingly, the worst performer is the standard Normal-GARCH model. For each criterion, the best model is among the MN-GARCH class. Furthermore, each of the chosen models is of the form $\operatorname{MN}(k, k)-$ GARCH, i.e., without suppression of any of the components' dynamics to a constant. When ranking according to the log-likelihood and the AIC, the top 5 models all belong to the MNGARCH class, whereas, according to the BIC, 4 of the 5, including the top three, belong to that class. All symmetric $\mathrm{MN}_{s}-\mathrm{GARCH}$ models perform relatively poorly. This is not surprising, given the pronounced negative skewness of the unconditional distribution.

In view of these results, the models $\mathrm{MN}(3,3)$ and $\mathrm{MN}(4,4)$, as well as $\mathrm{MN}(2,2)$ and $\mathrm{MN}(3,2)$, are retained for further consideration. The estimated parameter values of interest along with their approximate standard errors ${ }^{16}$ are shown in Table 2. (Due to the $\operatorname{GARCH}(1,1)$ specification we simply write $\beta$ for matrix $\beta_{1}$ and denote the typical element of $\beta$ by $\beta_{i j}$.) For comparison purposes, results for the standard Normal-GARCH model are also given.

In Table 2 , the components are ordered with respect to decreasing component means $\mu_{j}$,

\footnotetext{
${ }^{16}$ Standard errors were obtained by numerically computing the Hessian matrix at the ML estimates. The delta method was used to approximate the standard errors of functions of estimated quantities, namely, $\alpha_{1 i}+\beta_{i i}$, $i=1, \ldots, 4$, as well as the weights and means of the last component of each of the models.
} 
Table 1: Likelihood-based goodness of fit ${ }^{a}$

\begin{tabular}{|c|ccccccc|}
\hline Distributional & \multicolumn{4}{|c}{$L$} & \multicolumn{2}{c}{ AIC } & \multicolumn{2}{c|}{ BIC } \\
Model & $K$ & Value & Rank & Value & Rank & Value & Rank \\
\hline \hline Normal & 7 & -9142.8 & 16 & 18299.5 & 16 & 18348.1 & 16 \\
\hline $\mathrm{MN}(2,1)$ & 10 & -8962.7 & 15 & 17945.4 & 15 & 18014.8 & 15 \\
$\mathrm{MN}(3,1)$ & 13 & -8931.4 & 12 & 17888.7 & 14 & 17979.0 & 14 \\
$\mathrm{MN}(3,2)$ & 15 & -8857.5 & 4 & 17745.1 & 4 & 17849.2 & 2 \\
\hline $\mathrm{MN}(2,2)$ & 12 & -8872.5 & 5 & 17768.9 & 5 & 17852.4 & 3 \\
$\mathrm{MN}(3,3)$ & 17 & -8845.5 & 3 & 17725.0 & 3 & $\mathbf{1 7 8 4 3 . 1}$ & $\mathbf{1}$ \\
$\mathrm{MN}(4,4)$ & 22 & -8831.7 & 2 & $\mathbf{1 7 7 0 7 . 5}$ & $\mathbf{1}$ & 17860.3 & 5 \\
$\mathrm{MN}(5,5)$ & 27 & $-\mathbf{8 8 2 8 . 1}$ & $\mathbf{1}$ & 17710.2 & 2 & 17897.7 & 9 \\
\hline $\mathrm{MN}_{s}(2,2)$ & 11 & -8931.9 & 13 & 17885.7 & 13 & 17962.2 & 13 \\
$\mathrm{MN}_{s}(3,3)$ & 15 & -8908.5 & 10 & 17847.0 & 10 & 17951.2 & 12 \\
\hline Student's $t$ & 8 & -8932.7 & 14 & 17881.3 & 12 & 17936.9 & 11 \\
non-central $t t$ & 9 & -8908.2 & 9 & 17834.4 & 9 & 17896.9 & 8 \\
$t_{3}$ & 10 & -8884.3 & 6 & 17788.6 & 6 & 17858.1 & 4 \\
hyperbolic $^{\prime}$ & 9 & -8904.7 & 8 & 17827.4 & 8 & 17889.9 & 7 \\
EGB2 & 9 & -8895.0 & 7 & 17808.1 & 7 & 17870.6 & 6 \\
ADW & 9 & -8927.5 & 11 & 17873.1 & 11 & 17935.6 & 10 \\
\hline
\end{tabular}

$a_{\text {The leftmost column refers to the conditional distribution used with an } \mathrm{AR}(3)-}$ GARCH(1,1) model specification fitted for the NASDAQ returns. The column labeled $K$ refers to the number of parameters for the respective models; $L$ is the log likelihood; $\mathrm{AIC}=-2 L+2 K$ and $\mathrm{BIC}=-2 L+K \log T$. For each of the three criteria the criterion value and the ranking of the models are shown. Boldface entries indicate the best model for the particular criterion.

which also corresponds to an ordering with respect to increasing $\alpha_{1 j}$ (with the necessary exception of the third component of model $\mathrm{MN}(3,2)$ ), decreasing mixing weights, and a decreasing $\beta_{j j}$ (with the exception of components 1 and 2 in model $\mathrm{MN}(4,4)$ ). The results indicate a clear relationship between the component mean, $\mu_{j}$, and the component dynamics determined by $\alpha_{1 j}$ and $\beta_{j j}$. As $\mu_{j}$ drops, the increasing $\alpha_{1 j}$ reflects an increasing responsiveness to (negative) shocks, while there is more inertia in $\sigma_{j t}^{2}$ when shocks tend to be positive, as is reflected by the increasing values of $\beta_{j j}$.

Another striking result is that the volatility dynamics are stable in the sense that $\alpha_{1 j}+\beta_{j j}<$ 1 when $\mu_{j} \geq 0$ and unstable in the sense that $\alpha_{1 j}+\beta_{j j}>1$ for $\mu_{j}<0$. However, all estimated models themselves are stationary, as can be seen from the respective volatility persistence measures, $\rho_{\max }$, reported in the last row of Table 2 . This is due to the fact that the unstable components have sufficiently small mixing weights. In model $\mathrm{MN}(3,3)$, the first 
component is rather similar to the first component in model $\mathrm{MN}(2,2)$ and responds rather slowly to shocks. The second component, although just unstable $\left(\alpha_{12}+\beta_{22}=1.032\right)$, is more similar to the Normal-GARCH model and has an intermediate position. The third component, however, tends to heavily "overreact" to shocks, as reflected by the large value of $\alpha_{13}$; it is also characterized by a remarkably high value for constant $\alpha_{03}$ and is highly unstable, with $\alpha_{13}+\beta_{33}=1.870$. Observe also that, in each model with two or more components, the higher the volatility (as measured by the estimate of $\alpha_{1 i}+\beta_{i i}$ and the unconditional component variances $\left.\mathrm{E} \sigma_{i}^{2}, i=1, \ldots, 4\right)$, the lower is $\widehat{\mu}_{i}$, i.e., negative means arise in conjunction with higher variance. This finding is compatible with the well-known leverage effect (Black, 1976), which refers to the tendency for high volatility to coincide with negative returns (see, for example, Bekaert and $\mathrm{Wu}, 2000) .{ }^{17}$

The different responsiveness of the components to shocks is illustrated in Figure 2, which shows the square roots of the variance in the Normal-GARCH and of the component variances in model $\mathrm{MN}(3,3)$. The graphs clearly reveal the calm and rather hectic behavior of components 1 and 3 , respectively, while $\sigma_{2 t}$ mimics the evolution of $\sigma_{t}$ for the Normal-GARCH model. The relatively large constant $\alpha_{03}=0.332$ in component 3 is reflected in the floor of $\sigma_{3 t}$ at roughly 1 .

Returning to Table 2, the first two components of the $\operatorname{MN}(3,2)$ resemble those of the $\mathrm{MN}(2,2)$ model. With a component mean of $\mu_{3}=-2.281$ and the rather small weight of $\lambda_{3}=0.004$, the third component captures the large negative shocks and amounts to a jump process which does not include any conditional volatility dynamics. Model $\mathrm{MN}(4,4)$ is quite similar to model $\mathrm{MN}(3,3)$ but with the stable component with positive mean being split into two positive stable components.

Table 3 provides quantile values (13), skewness, kurtosis and the Jarque-Bera Lagrange multiplier test for normality for the "normalized" residuals, $\hat{v}_{t}=\Phi^{-1}\left(\hat{u}_{t}\right), t=1, \ldots, 7678$, of the four candidate $\mathrm{MN}$ and the symmetric $\mathrm{MN}_{s}$ models. The corresponding histograms, with one-at-a-time (i.e., not simultaneous) 95\% confidence intervals, and normal probability plots are displayed in Figure 4. The graph for $\mathrm{MN}(4,4)$ mimics that for $\mathrm{MN}(3,3)$ and is not shown.

The quantiles $U_{\xi}$ of the asymmetric MN models match the target values $\xi$ rather wellboth in the left and right tails - as can be seen from Table 3 and Figure 4. Note, however, that the left-tail fit of the $\operatorname{MN}(2,2)$ model is not as good as that for the models with $k>2$; this coincides with the preferences of the AIC and BIC criteria for higher parameterized MN models and is especially evident from the skewness and kurtosis statistics reported in Table 3.

\footnotetext{
${ }^{17} \mathrm{~A}$ number of GARCH models exist that incorporate an asymmetric relation between risk and return, e.g., the EGARCH of Nelson (1991) and the model of Glosten et al. (1993); see also Bollerslev et al. (1994).
} 
Table 2: MN-GARCH Parameter Estimates for NASDAQ Returns ${ }^{a}$

\begin{tabular}{|c|c|c|c|c|c|}
\hline & Normal & $\operatorname{MN}(2,2)$ & $\mathrm{MN}(3,3)$ & $\mathrm{MN}(3,2)$ & $\operatorname{MN}(4,4)$ \\
\hline$\alpha_{01}$ & $\begin{array}{c}0.014 \\
(0.0018)\end{array}$ & $\begin{array}{c}0.002 \\
(0.0008)\end{array}$ & $\begin{array}{c}0.000 \\
(0.0007)\end{array}$ & $\begin{array}{c}0.001 \\
(0.0008)\end{array}$ & $\begin{array}{c}0.003 \\
(0.0032)\end{array}$ \\
\hline$\alpha_{11}$ & $\begin{array}{c}0.117 \\
(0.0083)\end{array}$ & $\begin{array}{c}0.051 \\
(0.0066)\end{array}$ & $\begin{array}{c}0.022 \\
(0.0080)\end{array}$ & $\begin{array}{c}0.038 \\
(0.0068)\end{array}$ & $\begin{array}{c}0.067 \\
(0.0192)\end{array}$ \\
\hline$\beta_{11}$ & $\begin{array}{c}0.869 \\
(0.0089)\end{array}$ & $\begin{array}{c}0.920 \\
(0.0090)\end{array}$ & $\begin{array}{c}0.956 \\
(0.0137)\end{array}$ & $\begin{array}{c}0.934 \\
(0.0101)\end{array}$ & $\begin{array}{c}0.855 \\
(0.0461)\end{array}$ \\
\hline$\alpha_{11}+\beta_{11}$ & $\begin{array}{c}0.986 \\
(0.0032)\end{array}$ & $\begin{array}{c}0.971 \\
(0.0037)\end{array}$ & $\begin{array}{c}0.978 \\
(0.0063)\end{array}$ & $\begin{array}{c}0.972 \\
(0.0044)\end{array}$ & $\begin{array}{c}0.922 \\
(0.0345)\end{array}$ \\
\hline$\lambda_{1}$ & 1 & $\begin{array}{c}0.820 \\
(0.0255)\end{array}$ & $\begin{array}{c}0.541 \\
(0.0879)\end{array}$ & $\begin{array}{c}0.724 \\
(0.0427)\end{array}$ & $\begin{array}{c}0.373 \\
(0.1182)\end{array}$ \\
\hline$\mu_{1}$ & 0 & $\begin{array}{c}0.091 \\
(0.0100)\end{array}$ & $\begin{array}{c}0.164 \\
(0.0233)\end{array}$ & $\begin{array}{c}0.119 \\
(0.0133)\end{array}$ & $\begin{array}{c}0.200 \\
(0.0367)\end{array}$ \\
\hline $\mathrm{E} \sigma_{1}^{2}$ & 0.986 & 0.525 & 0.370 & 0.460 & 0.329 \\
\hline$\alpha_{02}$ & - & $\begin{array}{c}0.075 \\
(0.0235)\end{array}$ & $\begin{array}{c}0.012 \\
(0.0055)\end{array}$ & $\begin{array}{c}0.027 \\
(0.0122)\end{array}$ & $\begin{array}{c}0.000 \\
(0.0011)\end{array}$ \\
\hline$\alpha_{12}$ & - & $\begin{array}{c}0.512 \\
(0.0941)\end{array}$ & $\begin{array}{c}0.197 \\
(0.0425)\end{array}$ & $\begin{array}{c}0.379 \\
(0.0685)\end{array}$ & $\begin{array}{c}0.015 \\
(0.0054)\end{array}$ \\
\hline$\beta_{22}$ & - & $\begin{array}{c}0.727 \\
(0.0457)\end{array}$ & $\begin{array}{c}0.835 \\
(0.0260)\end{array}$ & $\begin{array}{c}0.768 \\
(0.0357)\end{array}$ & $\begin{array}{c}0.980 \\
(0.0071)\end{array}$ \\
\hline$\alpha_{12}+\beta_{22}$ & - & $\begin{array}{c}1.239 \\
(0.0588)\end{array}$ & $\begin{array}{c}1.031 \\
(0.0244)\end{array}$ & $\begin{array}{l}1.146 \\
(0.0413)\end{array}$ & $\begin{array}{c}0.995 \\
(0.0031)\end{array}$ \\
\hline$\lambda_{2}$ & 0 & $\begin{array}{c}0.180 \\
(0.0255)\end{array}$ & $\begin{array}{c}0.433 \\
(0.0832)\end{array}$ & $\begin{array}{c}0.272 \\
(0.0431)\end{array}$ & $\begin{array}{c}0.317 \\
(0.0743)\end{array}$ \\
\hline$\mu_{2}$ & - & $\begin{array}{l}-0.415 \\
(0.0575)\end{array}$ & $\begin{array}{l}-0.153 \\
(0.0548)\end{array}$ & $\begin{array}{l}-0.281 \\
(0.0508)\end{array}$ & $\begin{array}{c}0.035 \\
(0.0700)\end{array}$ \\
\hline $\mathrm{E} \sigma_{2}^{2}$ & - & 1.741 & 0.926 & 1.355 & 0.506 \\
\hline$\alpha_{03}$ & - & - & $\begin{array}{c}0.332 \\
(0.1913)\end{array}$ & $\begin{array}{c}0.825 \\
(0.6246)\end{array}$ & $\begin{array}{c}0.005 \\
(0.0086)\end{array}$ \\
\hline$\alpha_{13}$ & - & - & $\begin{array}{c}1.303 \\
(0.5179)\end{array}$ & - & $\begin{array}{c}0.246 \\
(0.0724)\end{array}$ \\
\hline$\beta_{33}$ & - & - & $\begin{array}{c}0.567 \\
(0.1389)\end{array}$ & - & $\begin{array}{c}0.824 \\
(0.0393)\end{array}$ \\
\hline$\alpha_{13}+\beta_{33}$ & - & - & $\begin{array}{c}1.870 \\
(0.4209)\end{array}$ & 0 & $\begin{array}{l}1.070 \\
(0.0416)\end{array}$ \\
\hline$\lambda_{3}$ & 0 & 0 & $\begin{array}{c}0.026 \\
(0.0111)\end{array}$ & $\begin{array}{c}0.004 \\
(0.0028)\end{array}$ & $\begin{array}{c}0.289 \\
(0.0841)\end{array}$ \\
\hline$\mu_{3}$ & - & - & $\begin{array}{l}-0.865 \\
(0.2223)\end{array}$ & $\begin{array}{l}-2.281 \\
(0.7251)\end{array}$ & $\begin{array}{c}-0.232 \\
(0.0824)\end{array}$ \\
\hline $\mathrm{E} \sigma_{3}^{2}$ & - & - & 2.936 & 0.825 & 0.974 \\
\hline$\alpha_{04}$ & - & - & - & - & $\begin{array}{c}0.373 \\
(0.2158)\end{array}$ \\
\hline$\alpha_{14}$ & - & - & - & - & $\begin{array}{c}1.427 \\
(0.6015)\end{array}$ \\
\hline$\beta_{44}$ & - & - & - & - & $\begin{array}{c}0.546 \\
(0.1524)\end{array}$ \\
\hline$\alpha_{14}+\beta_{44}$ & - & - & - & - & $\begin{array}{c}1.973 \\
(0.5002)\end{array}$ \\
\hline$\lambda_{4}$ & 0 & 0 & 0 & 0 & $\begin{array}{c}0.021 \\
(0.0089)\end{array}$ \\
\hline$\mu_{4}$ & - & - & - & - & $\begin{array}{c}-0.894 \\
(0.2412)\end{array}$ \\
\hline $\mathrm{E} \sigma_{4}^{2}$ & - & - & - & - & 2.941 \\
\hline$\rho_{\max }$ & 0.986 & 0.985 & 0.989 & 0.986 & 0.994 \\
\hline
\end{tabular}

${ }^{a}$ Standard errors are given in parentheses. Column $\mathrm{MN}(k, g)$ indicates the MN-GARCH $(1,1)$ with $k$ components, $g$ of which follow a GARCH process and $k-g$ components being restricted to having constant variances. $\mathrm{E} \sigma_{i}^{2}$, $i=1, \ldots, 4$, denotes the unconditional variance of component $i$, as computed from (8), and $\rho_{\max }$ is the measure of volatility persistence, that is, the largest eigenvalue of matrix (11). 
Evolution of $\sigma_{t}$ in the Normal-GARCH model
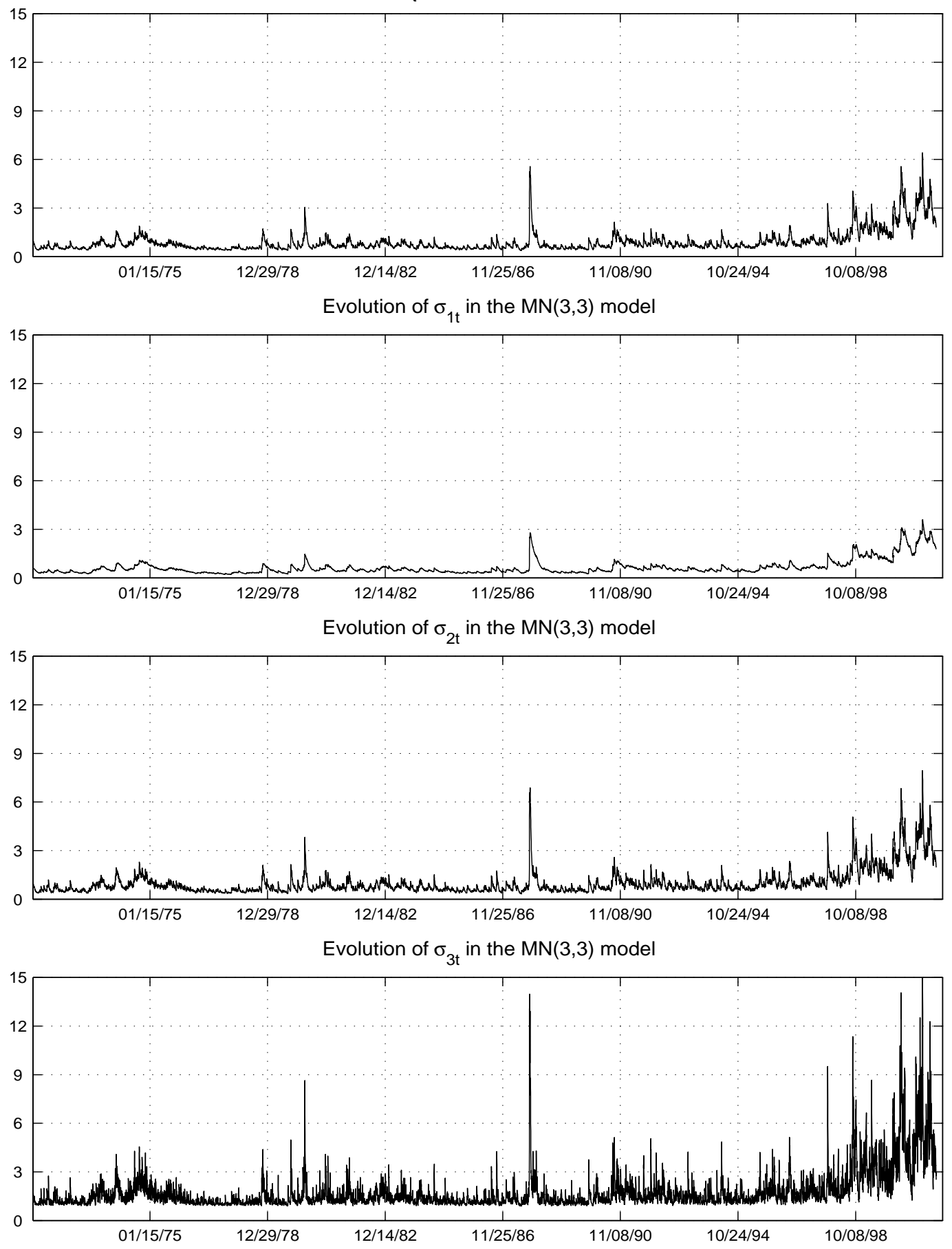

Figure 2: Volatility evolution for the Normal-GARCH and the MN(3,3)-GARCH models 
While there is no significant skewness and excess kurtosis in the "normalized" residuals of the $\mathrm{MN}(3,3)$ and $\mathrm{MN}(4,4)$ models, the model $\mathrm{MN}(2,2)$ fails to adequately capture these properties. However, all three models as well as the $\mathrm{MN}(3,2)$ pass the Pearson goodness-of-fit test at the $10 \%$ level. Note that the symmetric mixture models $\mathrm{MN}_{s}(2,2)$ and $\mathrm{MN}_{s}(3,3)$ are able to accommodate the excess kurtosis from the residuals, but clearly fail to capture the skewness. Taken altogether, it appears that the asymmetric diagonal-MN $(3,3)$ and diagonal-MN $(4,4)$ models provide an adequate description of the NASDAQ series.

Using (1) and (2), the conditional skewness $m_{3} / m_{2}^{3 / 2}$ of the fitted $\mathrm{MN}(3,3)$ model is shown in the top plot of Figure 3. Because of the increase in the conditional variance of the process towards the end of the data set, the implied skewness moves towards zero. The middle plots in the figure show the conditional density when the skewness reached its most extreme value of -1.56 . Its remarkable deviation from symmetry and the wide range of implied skewness values in the top plot emphasize the importance of time-varying skewness in this data set. The bottom plot shows the implied kurtosis, which appears to have a "natural lower bound" of 3 , which is explainable from (3) and the fact that $\hat{\mu}_{1}, \hat{\mu}_{2}$ and $\hat{\mu}_{3}$ are relatively close in value.

\subsection{Empirical Results for the Non-diagonal Models}

For the two-component full model, we obtain a triangular structure for $\beta$. The estimated model is of the form

$$
\left[\begin{array}{l}
\sigma_{1 t}^{2} \\
\sigma_{2 t}^{2}
\end{array}\right]=\left[\begin{array}{l}
0.002 \\
0.077
\end{array}\right]+\left[\begin{array}{l}
0.051 \\
0.538
\end{array}\right] \epsilon_{t-1}^{2}+\left[\begin{array}{ll}
0.918 & 0.000 \\
0.447 & 0.572
\end{array}\right]\left[\begin{array}{c}
\sigma_{1 t-1}^{2} \\
\sigma_{2 t-1}^{2}
\end{array}\right],
$$

with $\lambda=(0.806,0.194)^{T}$ and $\mu=(0.095,-0.395)^{T}$. The $\log$ likelihood, AIC and BIC of the model are -8869.4, 17764.8 and 17855.1, respectively. Thus, while AIC would prefer the full model, BIC prefers the diagonal specification.

Model (16) gives rise to a dynamic behavior similar to that of the diagonal model discussed in Section 4.4. For example, the volatility-persistence value is $\rho_{\max }=0.987$, which is close to the value 0.985 for the diagonal $\mathrm{MN}(2,2)$ in Table 2. Expression (B.4) shows how the components of the full model respond to innovations. The component-specific volatility persistence measures, given by $\alpha_{1 j}+\beta_{j j}$ in the diagonal case, are now computed as the Frobenius roots of matrices $\beta_{1}(1)+\alpha_{1}(1) e_{j}^{T}, j=1,2$. (Here, $e_{j}$ denotes the $j^{\text {th }} k \times 1$ unit vector.) These are 0.969 and 1.192, respectively, and are similar to those implied for the $\operatorname{MN}(2,2)$ model, namely, $\alpha_{11}+\beta_{11}=0.971$ and $\alpha_{12}+\beta_{22}=1.239$ (from line 4 in Table 2). Also, both processes give rise to quite similar unconditional variances. 

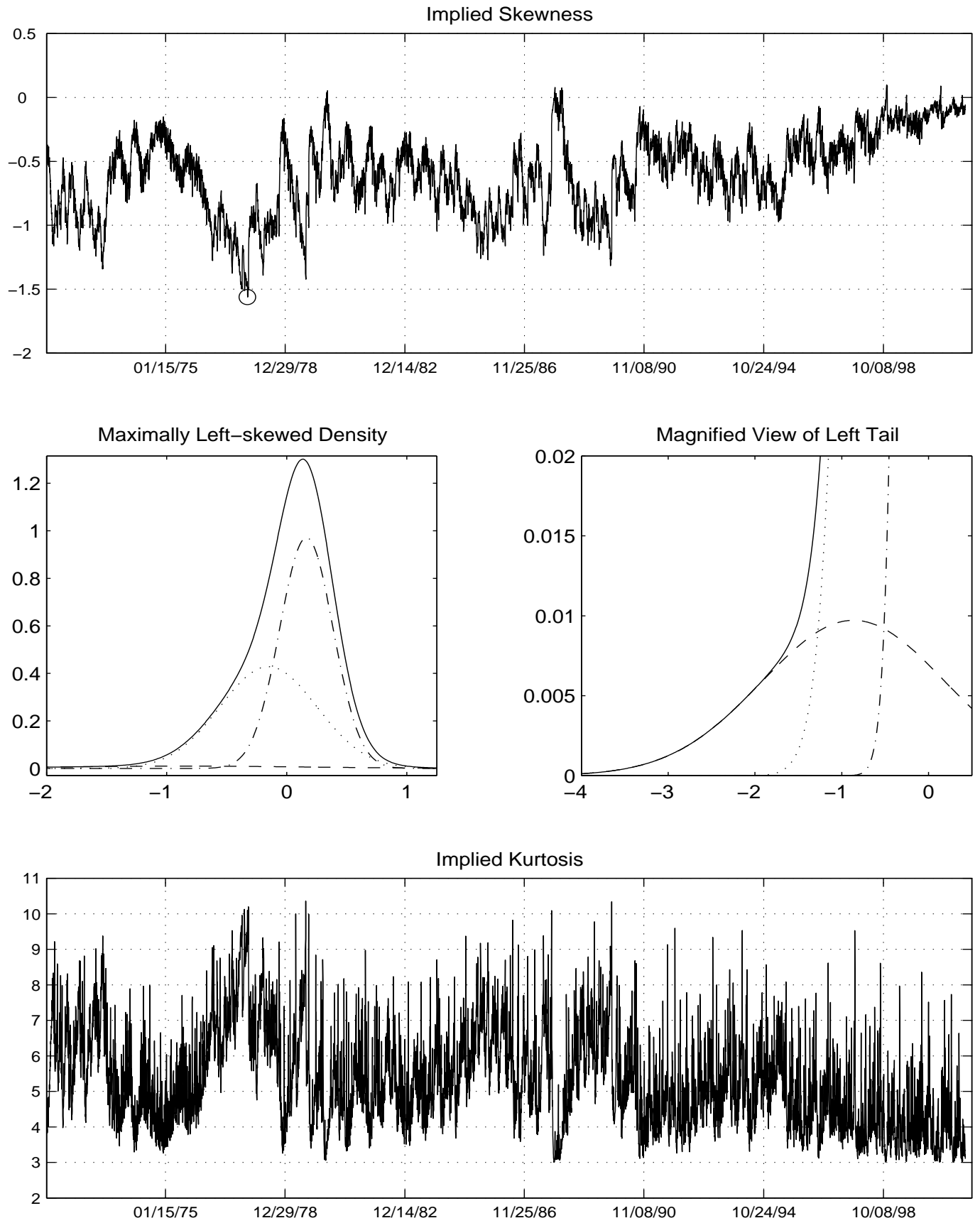

Figure 3: Top plot shows the implied skewness of fitted conditional densities for the NASDAQ data using the $\mathrm{MN}(3,3)$ model, with the inscribed circle indicating the maximal implied left skewness of -1.56 , the density (solid line) of which is plotted in the middle panel together with the weighted component densities (dashed, dotted and dash-dotted lines); the right graph in the middle panel is a magnification of the left tail. The bottom plot shows the implied kurtosis. 
The three-component full model is

$$
\left[\begin{array}{c}
\sigma_{1 t}^{2} \\
\sigma_{2 t}^{2} \\
\sigma_{3 t}^{2}
\end{array}\right]=\left[\begin{array}{l}
0.005 \\
0.000 \\
0.007
\end{array}\right]+\left[\begin{array}{l}
0.029 \\
0.283 \\
0.251
\end{array}\right] \epsilon_{t-1}^{2}+\left[\begin{array}{lll}
0.221 & 0.202 & 0.010 \\
1.572 & 0.235 & 0.001 \\
0.000 & 0.000 & 0.968
\end{array}\right]\left[\begin{array}{c}
\sigma_{1 t-1}^{2} \\
\sigma_{2 t-1}^{2} \\
\sigma_{3 t-1}^{2}
\end{array}\right],
$$

with $\lambda=(0.622,0.372,0.006)^{T}, \mu=(0.145,-0.220,-1.375)^{T}$, and $\rho_{\max }=0.994$. The $\log -$ likelihood is -8842.4 , which is a negligible improvement compared to the diagonal model.

\subsection{Extension to Fat-Tailed Components: The Mixed- - -GARCH}

An extension of the MN-GARCH model which very naturally suggests itself is to replace the normal distribution with a fatter-tailed alternative. This would, in the NASDAQ case, help to accomodate the lack of fit of the $\mathrm{MN}(2,2)-\operatorname{GARCH}(1,1)$ and potentially render unnecessary the $\mathrm{MN}(3,3)$ model, also resulting in a more parsimonious model. In this case, the component densities are characterized by an additional shape parameter, which may or may not differ across the components. As the conditional variance of $\epsilon_{t}$ is affected by this shape parameter, we have

$$
\mathrm{E}\left[\epsilon_{t}^{2} \mid \Psi_{t-1}\right]=\sum_{j=1}^{k} \lambda_{j} \kappa_{j} \sigma_{j t}^{2}+c
$$

where $c$ is as in (8) and $\kappa_{j}$ is a function of the shape parameter of the $j^{\text {th }}$ component. For example, if the component densities are Student's $t$ with $\nu_{j}$ degrees of freedom, $j=1, \ldots, k$, then $\kappa_{j}=\nu_{j} /\left(\nu_{j}-2\right)$. Straightforward calculations show that the stationarity condition (9) is easily extended, with the vector of mixing weights $\lambda$ being replaced by $\lambda \odot \kappa$, where $\odot$ denotes element-by-element multiplication (the Hadamard product) of conformable matrices and $\kappa=\left[\kappa_{1}, \ldots, \kappa_{k}\right]^{T}$.

Using a mixture of Student's $t$ distributions for the NASDAQ, first consider the same degrees of freedom parameter, $\nu$, for each mixture component, jointly estimated with the remaining parameters. The resulting model then generalizes that proposed by Neely (1999) who used the Student's $t$ with the Vlaar and Palm (1993) model. In the two-component case, $\hat{\nu}=14.8$ (with standard error 3.4), indicating a relatively mild deviation from normality. With log likelihood value -8862.4 and 13 parameters, this resulted in a AIC value of 17750.9 and a BIC value of 17841.2 , i.e., the $\mathrm{AIC}$ favors the $\mathrm{MN}(3,3)-\operatorname{GARCH}(1,1)$ formulation, while the BIC is virtually indifferent. Thus, the introduction of the fatter-tailed density cannot replace the added dynamics which are allowed for in the $\mathrm{MN}(3,3)$ case.

Allowing the degrees of freedom to differ in this model, the low-volatility component has $\hat{\nu}_{1}=44.2$ (with "standard error" 47.9), and the high volatility unstationary component has 
$\hat{\nu}_{2}=8.63$ (with standard error 1.81). The log likelihood of this model with 14 parameters is -8857.9, implying AIC $=17743.8$ and $\mathrm{BIC}=17841.0$. Compared to the $\mathrm{MN}(3,3)$ model, the discussion of the former paragraph still applies.

Using the Student's $t$ in the three component model (with equal degrees of freedom) resulted in $\hat{\nu}=109.8$ (with the meaningless standard error of 310) and log likelihood value -8845.1 , clearly demonstrating the adequacy of the normal.

It must be emphasized that these results are based on a single data set; the "mixed- $t$ GARCH" might indeed be useful in other contexts. However, its use ventures into the ad-hoc realm which we have endeavored to avoid.

Table 3: In-sample Fit of AR(3)-GARCH(1,1) MN Models ${ }^{a}$

\begin{tabular}{|c|lllllll|}
\hline & Normal & $\mathrm{MN}_{\mathrm{s}}(2,2)$ & $\mathrm{MN}_{\mathrm{s}}(3,3)$ & $\mathrm{MN}(2,2)$ & $\mathrm{MN}(3,2)$ & $\mathrm{MN}(3,3)$ & $\mathrm{MN}(4,4)$ \\
\hline$U_{0.1}$ & 0.703 & 0.234 & 0.182 & 0.195 & 0.143 & 0.130 & 0.130 \\
$U_{0.5}$ & 1.420 & 0.755 & 0.821 & 0.495 & 0.612 & 0.560 & 0.560 \\
$U_{1}$ & 1.954 & 1.511 & 1.719 & 0.886 & 1.042 & 1.094 & 1.055 \\
$U_{2.5}$ & 3.647 & 3.829 & 3.985 & 2.370 & 2.527 & 2.761 & 2.748 \\
$U_{5}$ & 5.978 & 6.851 & 6.981 & 5.522 & 5.548 & 5.731 & 5.640 \\
$U_{10}$ & 9.820 & 11.64 & 11.66 & 10.78 & 10.68 & 10.51 & 10.63 \\
$U_{90}$ & 93.01 & 91.63 & 91.61 & 90.49 & 90.41 & 90.28 & 90.10 \\
$U_{95}$ & 96.69 & 96.20 & 96.26 & 95.29 & 95.26 & 95.08 & 95.21 \\
$U_{97.5}$ & 98.41 & 98.50 & 98.42 & 97.67 & 97.64 & 97.60 & 97.51 \\
$U_{99}$ & 99.30 & 99.65 & 99.57 & 99.05 & 99.04 & 99.01 & 99.05 \\
$U_{99.5}$ & 99.69 & 99.88 & 99.83 & 99.58 & 99.51 & 99.49 & 99.49 \\
$U_{99.9}$ & 99.90 & 99.99 & 99.99 & 99.91 & 99.88 & 99.91 & 99.92 \\
\hline$X^{2}$ & 0.000 & 0.000 & 0.000 & 0.276 & 0.168 & 0.186 & 0.325 \\
Skewness & $-0.672^{* * *}$ & $-0.298^{* * *}$ & $-0.290^{* * *}$ & $-0.088^{* * *}$ & $-0.054^{*}$ & -0.046 & -0.037 \\
Kurtosis & $2.521^{* * *}$ & 0.064 & 0.027 & $0.134^{* *}$ & $0.094^{*}$ & 0.011 & -0.012 \\
JB & $2609.7^{* * *}$ & $115.1^{* * *}$ & $108.1^{* * *}$ & $15.6^{* * *}$ & $6.5^{* *}$ & 2.7 & 1.8 \\
\hline
\end{tabular}

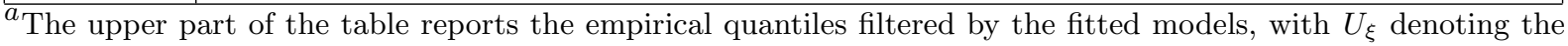
$\xi \%$-quantile. The lower part reports test results on the distributional properties of the transformed residuals. $X^{2}$ refers to the $p$-value of the Pearson goodness-of-fit test (14) after transformation (12), with $g=100$ and $g-K-1$ degrees of freedom, where $K$ is the number of parameters of the respective models. The last three rows are based on transformation (15). "Skewness" denotes the coefficient of skewness $\gamma_{1}=m_{3} / m_{2}^{3 / 2}$ and "Kurtosis" the coefficient of excess kurtosis $\gamma_{2}-3=m_{4} / m_{2}^{2}-3$. Under normality, $T \gamma_{1}^{2} / 6 \sim \chi^{2}(1)$ and $T\left(\gamma_{2}-3\right)^{2} / 24 \sim \chi^{2}(1)$ asymptotically. JB is the value of the Jarque-Bera (1987) Lagrange multiplier test for normality, i.e., JB $=T \gamma_{1}^{2} / 6+T\left(\gamma_{2}-3\right)^{2} / 24$ (cf. Lütkepohl, 1991, pp. 152-156). Asterisks ${ }^{*},{ }^{* *}$ and ${ }^{* * *}$ indicate significance at the $10 \%, 5 \%$ and $1 \%$ levels, respectively. 

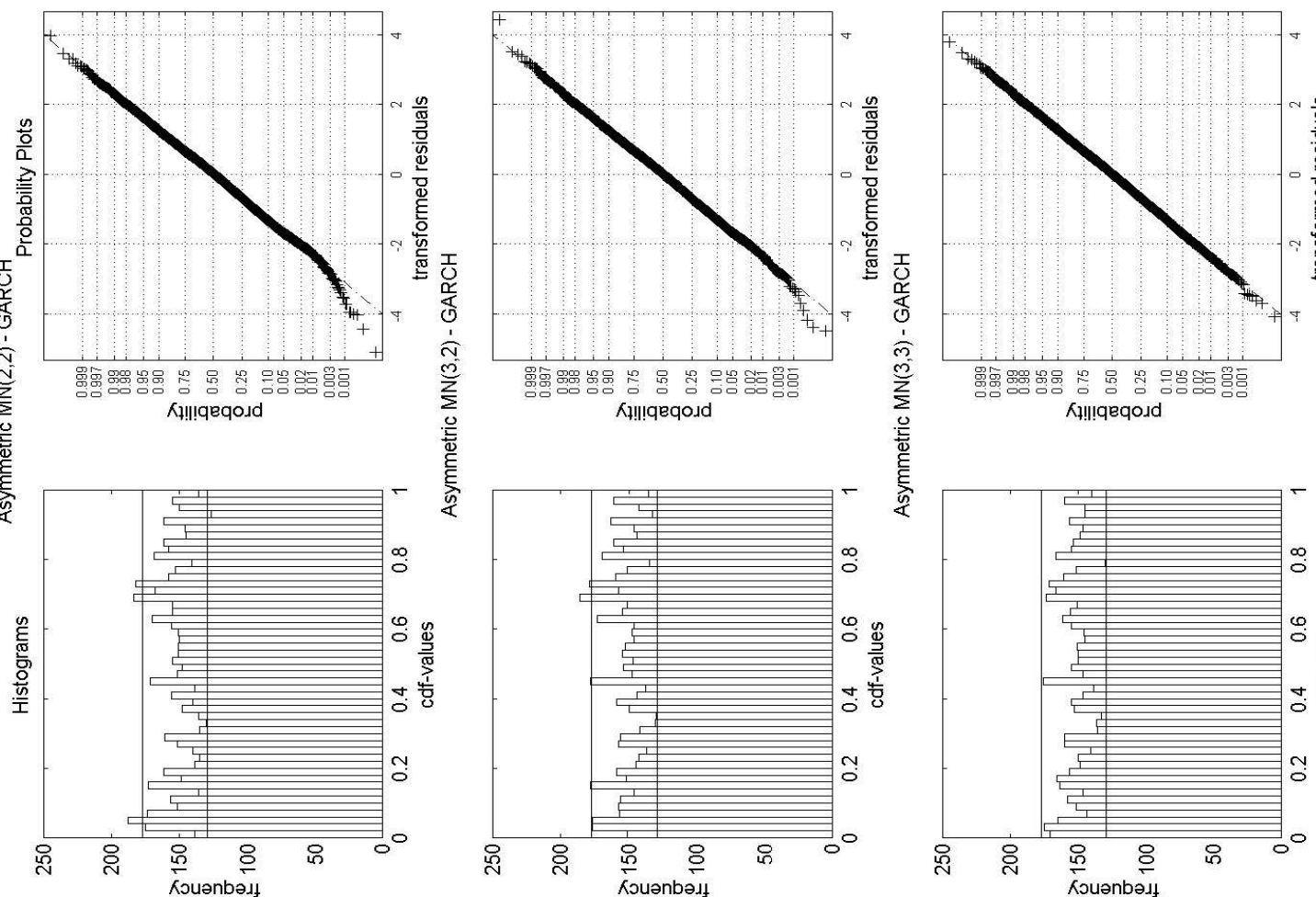

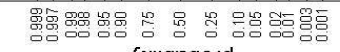

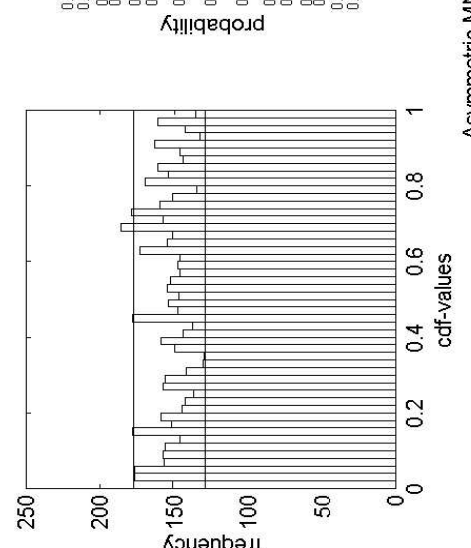

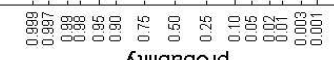
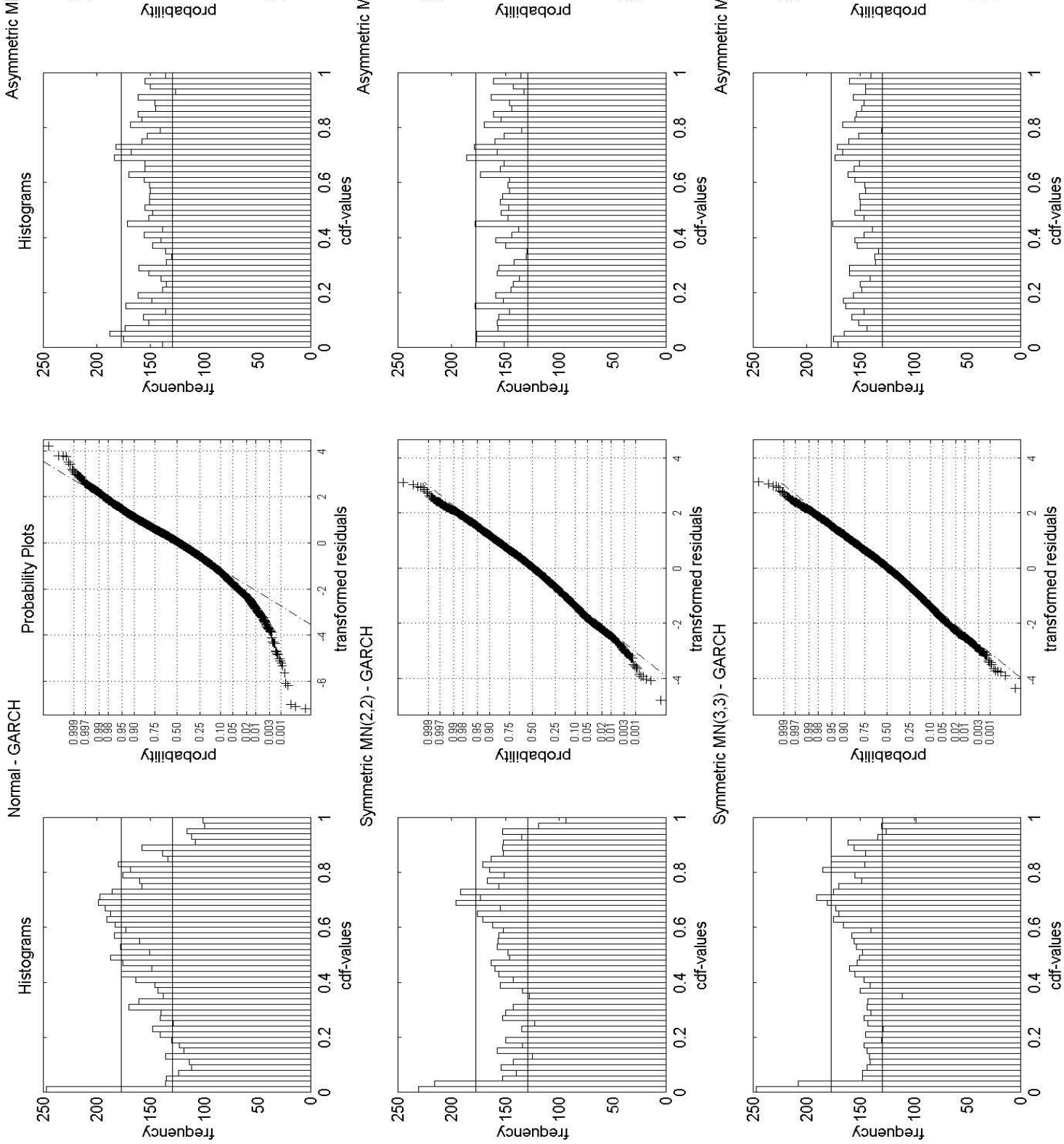

$\frac{\infty}{\pi}$

‡

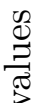

tैํㅇ

$\underset{+}{+}$

n 


\section{Conclusions}

We have investigated the properties and the usefulness of a class of conditionally heteroskedastic models for financial return series which re-employs the normality assumption via a mixed normal structure. The model gives rise to rich dynamics including time-varying skewness and kurtosis, which is otherwise not encountered in GARCH models driven by innovations from the "usual" asymmetric fat-tailed distributions. When applied to the returns on the NASDAQ index, the model class fairs extremely well compared to commonly used competing distributional specifications. Moreover, it offers a disaggregation of the conditional variance process which is amenable to economic interpretation, including the well-known leverage effect.

There are several possible generalizations of the proposed model which might be worth future investigation. First, allowing for time-varying mixture weights, as proposed in Vlaar and Palm (1993) and implemented, for example, in Beine and Laurent (1999) to model exchange rates, with the weights depending on central bank interventions. Second, more general, asymmetric GARCH structures, such as those proposed by Ding et al. (1993); and Sentana (1995), could be entertained. Third, the use of a weighted likelihood function, as employed in Mittnik and Paolella (2000) for achieving better out-of-sample forecasting performance, might also prove useful in this context. Finally, models with more general dynamics in the mean equation might be advantageous for modeling certain nonlinear time series. To this end, Wong and $\operatorname{Li}(2001)$ proposed a mixture autoregressive $\operatorname{ARCH}\left(k ; p_{1}, \ldots, p_{k} ; q_{1}, \ldots, q_{k}\right)$ model, which allows for rather general mean dynamics. It is defined by

$$
F\left(y_{t} \mid \Psi_{t-1}\right)=\sum_{j=1}^{k} \lambda_{j} \Phi\left(\frac{\epsilon_{j, t}}{\sigma_{j, t}}\right),
$$

where $\epsilon_{j, t}=y_{t}-\gamma_{j, 0}-\sum_{i=1}^{p_{j}} \gamma_{j, i} y_{t-i}$ and $\sigma_{j, t}^{2}=\alpha_{j, 0}+\sum_{i=1}^{q_{j}} \alpha_{j, i} \epsilon_{j, t-i}^{2}$. In modeling asset returns, however, the benefits of additional efforts in modeling the mean dynamics tend to be negligible, so that specification (5) may be preferable in the present context. Furthermore, in contrast to (17), the model structure adapted here allows a clear separation between the dynamics in the mean and in volatility, and, moreover, it leads to tractable stationarity conditions with insightful interpretations. ${ }^{18}$

\footnotetext{
${ }^{18}$ In (17), the dynamics in the means also account for conditional heteroskedasticity. The interaction between $\mathrm{AR}-$ and ARCH-dynamics leads to rather complicated stationarity conditions, especially for autoregressive orders exceeding one.
} 


\section{Appendix}

\section{A Computation of Constant $c$ in (8)}

For $Y \sim \operatorname{MN}\left(\lambda_{1}, \ldots, \lambda_{k}, \mu_{1}, \ldots, \mu_{k}, \sigma_{1}^{2}, \ldots, \sigma_{k}^{2}\right)$, we have

$$
\operatorname{Var}[Y]=\sum_{j=1}^{k} \lambda_{j} \sigma_{j}^{2}+\sum_{j=1}^{k} \lambda_{j} \mu_{j}^{2}-\left(\sum_{j=1}^{k} \lambda_{j} \mu_{j}\right)^{2}=\sum_{j=1}^{k} \lambda_{j} \sigma_{j}^{2}+c .
$$

Hence, with $\mathrm{E}[Y]=\sum_{j=1}^{k} \lambda_{j} \mu_{j}=0 \Leftrightarrow \mu_{k}=-\sum_{j=1}^{k-1} \frac{\lambda_{j}}{\lambda_{k}} \mu_{j}$,

$$
\begin{aligned}
c & =\sum_{j=1}^{k} \lambda_{j} \mu_{j}^{2}=\sum_{j=1}^{k-1} \lambda_{j} \mu_{j}^{2}+\lambda_{k}\left(\sum_{j=1}^{k-1} \frac{\lambda_{j}}{\lambda_{k}} \mu_{j}\right)^{2} \\
& =\frac{1}{\lambda_{k}}\left(\sum_{j=1}^{k-1} \lambda_{j} \mu_{j}^{2}\left(1-\sum_{j=1}^{k-1} \lambda_{j}\right)+\sum_{j=1}^{k-1} \sum_{r=1}^{k-1} \lambda_{j} \lambda_{r} \mu_{j} \mu_{r}\right) \\
& =\frac{1}{\lambda_{k}}\left(\sum_{j=1}^{k-1} \lambda_{j} \mu_{j}^{2}-\sum_{j=1}^{k-1} \lambda_{j} \mu_{j}^{2} \sum_{r=1}^{k-1} \lambda_{r}+\sum_{j=1}^{k-1} \sum_{r=1}^{k-1} \lambda_{j} \lambda_{r} \mu_{j} \mu_{r}\right) \\
& =\frac{1}{\lambda_{k}}\left(\sum_{j=1}^{k-1} \lambda_{j} \mu_{j}^{2}-\sum_{j=1}^{k-1} \lambda_{j} \mu_{j}^{2} \sum_{r \neq j} \lambda_{r}+2 \sum_{j<r<k} \sum_{j} \lambda_{r} \mu_{j} \mu_{r}\right) \\
& =\frac{1}{\lambda_{k}}\left(\sum_{j=1}^{k-1} \lambda_{j} \mu_{j}^{2}-\sum_{j<r<k} \sum_{j} \lambda_{j} \lambda_{r}\left(\mu_{j}-\mu_{r}\right)^{2}\right) .
\end{aligned}
$$

For $k=2$, the expression for constant $c$ reduces to $c=\frac{\lambda_{1}}{1-\lambda_{1}} \mu_{1}^{2}$.

\section{B Derivation of Stationarity Condition (9)}

By deriving a GARCH equation for the conditional variance of $\epsilon_{t}$,

$$
\mathrm{E}\left(\epsilon_{t}^{2} \mid \Psi_{t-1}\right)=\lambda^{T} \sigma_{t}^{(2)}+c
$$

we show that the process is weakly stationary if the eigenvalues of matrix $\Phi$, defined by (11), are less than one in absolute value or, equivalently, if the roots of the characteristic equation

$$
\operatorname{det}\left[I-\alpha(z) \lambda^{T}-\beta(z)\right]=0
$$

are outside the unit circle. By use of the non-negativity conditions for the $\alpha_{i}$ and $\beta_{i}$, this is equivalent to condition (9).

Consider the MN-GARCH process (6). Using the fact that, for any invertible matrix $C$, $C^{-1}=C^{+} / \operatorname{det} C$, where $C^{+}$denotes the adjoint matrix of $C,(6)$ can be written as

$$
\operatorname{det}[I-\beta(L)] \sigma_{t}^{(2)}=[I-\beta(1)]^{+} \alpha_{0}+[I-\beta(L)]^{+} \alpha(L) \epsilon_{t}^{2} .
$$


Without loss of generality, it can be assumed that the $\operatorname{roots}$ of $\operatorname{det}[I-\beta(z)]=0$ lie outside the unit circle, since otherwise the non-stochastic part of difference equation (5) would be explosive.

The construction of the adjoint matrix implies that

$$
[I-\beta(L)]^{+} \alpha(L)=\left[\begin{array}{c}
\operatorname{det} B(L)_{1} \\
\operatorname{det} B(L)_{2} \\
\vdots \\
\operatorname{det}(L)_{k}
\end{array}\right]
$$

where $B(L)_{j}$ is matrix $I-\beta(L)$ with the $j^{\text {th }}$ column being replaced by $\alpha(L)$. Thus, (B.1) gives rise to $k$ univariate equations of the form

$$
\operatorname{det}[I-\beta(L)] \sigma_{t j}^{2}=\left(\operatorname{det} B(L)_{j}\right) \epsilon_{t}^{2}+\operatorname{det} A(1)_{j}, \quad j=1, \ldots, k,
$$

where $A(1)_{j}$ is matrix $I-\beta(1)$ with the $j^{\text {th }}$ column being replaced by $\alpha_{0}$.

Note that (B.3) can not be interpreted as a GARCH equation for $\sigma_{j t}^{2}$, because $\sigma_{j t}^{2}$ is not the conditional variance of $\epsilon_{t}$. If such an interpretation were correct, weak stationarity would require the roots of

$$
\operatorname{det}[I-\beta(z)]-\operatorname{det} B(z)_{j}=\operatorname{det}\left[I-\beta(z)-\alpha(z) e_{j}^{T}\right]=0, \quad j=1, \ldots, k,
$$

to be outside the unit circle, where $e_{j}$ is the $j^{\text {th }}$ unit vector in $\mathbb{R}^{k}{ }^{19}$ The conditional variance of $\epsilon_{t}$ is given by a linear combination of the conditional component variances, i.e.,

$$
\mathrm{E}\left[\epsilon_{t}^{2} \mid \Psi_{t-1}\right]=\sigma_{t}^{2}=\sum_{j=1}^{k} \lambda_{j} \sigma_{j t}^{2}+c .
$$

The variance of the process $\left\{\epsilon_{t}\right\}$ thus follows a univariate GARCH equation,

$$
\operatorname{det}[I-\beta(L)] \sigma_{t}^{2}=\left(\sum_{j=1}^{k} \lambda_{j} \operatorname{det} B(L)_{j}\right) \epsilon_{t}^{2}+c^{*},
$$

where $c^{*}=\sum_{j} \lambda_{j} A(1)_{j}+\operatorname{det}(I-\beta(1)) c=c \operatorname{det}\left[I-\beta(1)+\alpha_{0} \lambda^{T} / c\right]$ is constant. The argument is completed by following the same lines as in Gourieroux (1997, p. 37). Defining $w_{t}=$ $\epsilon_{t}^{2}-\sigma_{t}^{2}$ and replacing, in (B.5), $\sigma_{t}^{2}$ by $\epsilon_{t}^{2}-w_{t}$, we obtain an $\operatorname{ARMA}(\max \{p k, p(k-1)+q\}, p k)$ representation for the $\epsilon_{t}^{2}$ process,

$$
\left[\operatorname{det}(I-\beta(L))-\sum_{j=1}^{k} \lambda_{j} \operatorname{det} B(L)_{j}\right] \epsilon_{t}^{2}=\operatorname{det}[I-\beta(L)] w_{t}+c^{*} .
$$

\footnotetext{
${ }^{19}$ The first equality in (B.4) follows directly from the linearity of $\operatorname{det}(\cdot)$ in columns.
} 
Hence, the sequence $\mathrm{E}\left(\epsilon_{t}^{2}\right)$ converges and the process $\left\{\epsilon_{t}^{2}\right\}$ is weakly stationary if the roots of the characteristic equation ${ }^{20}$

$$
\begin{aligned}
\operatorname{det}[I-\beta(z)]-\sum_{j=1}^{k} \lambda_{j} \operatorname{det} B(z)_{j} & =\operatorname{det}\left[I-\beta(z)-\alpha(z) \lambda^{T}\right] \\
& =\sum_{j=1}^{k} \lambda_{j} \operatorname{det}\left[I-\beta(z)-\alpha(z) e_{j}^{T}\right] \\
& =0
\end{aligned}
$$

are larger than unity or, equivalently, the spectral radius, $\rho(\cdot)$, of the transition matrix (11) satisfies $\rho(\Phi)<1 .^{21}$ If $\rho(\Phi)<1$, then (9) holds, and, by the non-negativity of $\Phi$, guarantees the required positivity in $(8) .{ }^{22}$

Next, assume that $\operatorname{det}\left[I-\alpha(1) \lambda^{T}-\beta(1)\right]>0$ and note that, by the Frobenius Theorem (Gantmacher, 1959, p. 66), the largest root in magnitude of $\Phi$ is real and non-negative, so it suffices to show that the determinant condition implies that there is no real root of $\Phi$ equal to or larger than one. Define, analogous to $\Phi$, the matrix

$$
B=\left[\begin{array}{ccccc}
\beta_{1} & \beta_{2} & \cdots & \beta_{r-1} & \beta_{r} \\
I & 0 & \cdots & 0 & 0 \\
0 & I & & 0 & 0 \\
\vdots & \vdots & \ddots & \vdots & \vdots \\
0 & 0 & \cdots & \dot{I} & 0
\end{array}\right],
$$

where $r=\max \{p, q\}$. As was mentioned above, it can be assumed without loss of generality that the eigenvalues of $B$ are inside the unit circle, i.e., $\operatorname{det}[I-\beta(z)]=0 \Rightarrow|z|>1$. From (B.6), the characteristic equation of matrix $\Phi$ is

$$
\begin{aligned}
\operatorname{det}(z I-\Phi) & =\operatorname{det}\left(z^{r} I-\sum_{i=1}^{p} \beta_{i} z^{r-i}-\sum_{i=1}^{q} \alpha_{i} \lambda^{T} z^{r-i}\right) \\
& =\operatorname{det}(z I-B)\left[1-\lambda^{T}\left(z^{r} I-\sum_{i=1}^{p} \beta_{i} z^{r-i}\right)^{-1} \sum_{i=1}^{q} \alpha_{i} z^{r-i}\right] \\
& =\operatorname{det}(z I-B)\left[1-\lambda^{T}\left(I-\sum_{i=1}^{p} \beta_{i} z^{-i}\right)^{-1} \sum_{i=1}^{q} \alpha_{i} z^{-i}\right] .
\end{aligned}
$$

From non-negativity, $\sum_{i=1}^{q} \alpha_{i} z^{-i}$ monotonically decreases in $z .\left(I-\sum_{i=1}^{p} \beta_{i} z^{-i}\right)^{-1}$ forms the first $k$ rows and columns of $\left(I-B z^{-1}\right)^{-1}=\sum_{i=0}^{\infty} B^{i} z^{-i} \geq 0$ for $z>\rho(B)$. It decreases

\footnotetext{
${ }^{20}$ Recall that a GARCH process is serially uncorrelated; hence the process is weakly stationary if the variance exists.

${ }^{21}$ The first equality can be obtained by repeated use of the linearity of $\operatorname{det}(\cdot)$ in columns. It is, however, a direct consequence of the Sherman-Morrison formula for determinants, stating that, for matrix A and vectors $u$ and $v, \operatorname{det}\left(A+u v^{T}\right)=\operatorname{det} A+v^{T} A^{+} u$ (see, e.g., Henderson and Searle, 1981).

${ }^{22}$ It is well-known (see, e.g., Bowden, 1972), that $\left[I-\alpha(z) \lambda^{T}-\beta(z)\right]^{-1}$ is the upper left block of matrix $(I-\Phi z)^{-1} \geq 0$ for $z^{-1}>\rho(\Phi)$.
} 
monotonically in $z$. Hence, it follows that, if $\operatorname{det}(I-\Phi)=\operatorname{det}\left[I-\alpha(1) \lambda^{T}-\beta(1)\right]>0$, then $\rho(\Phi)<1$.

To appreciate the stationarity condition of MN-GARCH processes, note that, combining (B.6) with (B.3) shows that stability does not require the condition $\operatorname{det}\left(I-\beta(1)-\alpha(1) e_{j}^{T}\right)$ $>0$ to hold for each of the $k$ relationships in (B.3). It suffices that positivity holds for the weighted average with weights $\lambda_{j}, j=1, \ldots, k$.

\section{References}

Aitkin, M., Anderson, D., and Hinde, J. (1981). Statistical Modelling of Data on Teaching Styles (with discussion). Journal of the Royal Statistical Society A, 144:419-461.

Akaike, H. (1973). Information Theory and an Extension of the Maximum Likelihood Principle. In Petrov, B. and Csaki, F., editors, 2nd International Symposium on Information Theory, 267-281, Akademiai Kiado, Budapest.

Akgiray, V. and Booth, G. G. (1987). Compound Distribution Models of Stock Returns: An Empirical Comparison. The Journal of Financial Research, 10:269-280.

Ball, C. A. and Torous, W. N. (1983). A Simplified Junp Process for Common Stock Returns. Journal of Financial and Quantitative Analysis, 18:53-65.

Bauwens, L., Bos, C. S., and van Dijk, H. K. (1999a). Adaptive Polar Sampling with an Application to Bayes Measure of Value-at-Risk. Tinbergen Institute Discussion Paper TI 99-082/4, Erasmus University Rotterdam.

Bauwens, L., Lubrano, M., and Richard, J.-M. (1999b). Bayesian Inference in Dynamic Econometric Models. Oxford University Press, New York.

Beine, M. and Laurent, S. (1999). Central Bank Interventions and Jumps in Double Long Memory Models of Daily Exchange Models. Paper presented at the annual Helenau Workshop, Humboldt University, Berlin.

Bekaert, G. and Wu, G. (2000). Asymmetric Volatility and Risk in Equity Markets. The Review of Financial Studies, 13:1-42.

Berkowitz, J. (2001). Testing Density Forecasts, with Applications to Risk Management. Journal of Business and Economic Statistics, 19:465-474.

Black, F. (1976). Studies of Stock Market Volatility Changes. In Proceedings of the American Statistical Association, Business and Economic Statistics Section, 177-181.

Bollerslev, T. (1986). Generalized Autoregressive Conditional Heteroskedasticity. Journal of Econometrics, 31:307-327.

Bollerslev, T. (1987). A Conditionally Heteroskedastic Time Series Model For Speculative Prices and Rates of Returns. The Review of Economics and Statistics, 69:542-547.

Bollerslev, T., Engle, R. F., and Nelson, D. B. (1994). ARCH Models. In Engle, R. and McFadden, D., editors, Handbook of Econometrics, Vol. 4, Chapter 49. Elsevier Science B.V., Amsterdam, The Netherlands.

Bowden, R. (1972). The Generalized Characteristic Equation of a Linear Dynamic System. Econometrica, 40:201-203.

Brännäs, K. and Nordman, N. (2001). Conditional Skewness Modelling for Stock Returns. Umeå Economic Studies 562, Department of Economics, Umeå University, Sweden. 
Chen, H., Cheng, J., and Kalbfleisch, J. D. (2001). A Modified Likelihood Ratio Test for Homogeneity in Finite Mixture Models. Journal of the Royal Statistical Society, 63:1929.

Chib, S. and Greenberg, E. (1996). Markov Chain Monte Carlo Simulation Methods in Econometrics. Econometric Theroy, 12:409-431.

Dacco, R. and Satchell, S. (1999). Why do Regime-Switching Models Forecast so Badly? Journal of Forecasting, 18:1-16.

Dasgupta, A. and Raftery, A. E. (1998). Detecting Features in Spatial Point Processes With Clutter via Model-Based Clustering. Journal of the American Statistical Association, 93:294-302.

Dempster, A. P., Laird, N. M., and Rubin, D. B. (1977). Maximum Likelihood from Incomplete Data via the EM Algorithm. Journal of the Royal Statistical Society B, 39:1-38.

Diebold, F. X., Gunther, T. A., and Tay, A. S. (1998). Evaluating Density Forecasts with Applications to Financial Risk Management. International Economic Review, 39:863883.

Ding, Z., Granger, C. W. J., and Engle, R. F. (1993). A Long Memory Property of Stock Market Returns and a New Model. Journal of Empirical Finance, 1:83-106.

Dudewicz, E. J. and Mishra, S. N. (1988). Modern Mathematical Statistics. John Wiley \& Sons, New York.

Dueker, M. J. (1997). Markov Switching in GARCH Processes and Mean-Reverting StockMarket Volatility. Journal of Business and Economic Statistics, 15:26-34.

Eberlein, E. and Keller, U. (1995). Hyperbolic Distributions in Finance. Bernoulli, 1:281-299.

Everitt, B. S. and Hand, D. J. (1981). Finite Mixture Distributions. Chapman and Hall, London.

Fama, E. (1965). The Behavior of Stock Market Prices. Journal of Business, 38:34-105.

Feng, Z. D. and McCulloch, C. E. (1996). Using Bootstrap Likelihood Ratios in Finite Mixture Models. Journal of the Royal Statistical Society, 58:609-617.

Fraley, C. and Raftery, A. E. (1998). How Many Clusters? Which Clustering Method? Answers via Model-Based Cluster Analysis. The Computer Journal, 41:578-588.

Francq, C., Roussignol, M., and Zakoïan, J.-M. (2001). Conditional Heteroskedasticity Driven by Hidden Markov Chains. Journal of Time Series Analysis, 22:197-220.

Franses, P. H. and van Dijk, D. (1996). Forecasting Stock Market Volatility Using (Non-Linear) GARCH Models. Journal of Forecasting, 15:229-235.

Friend, W. E. and Westerfield, R. (1980). Co-skewness and Capital Asset Pricing. Journal of Finance, 35:897-914.

Gantmacher, F. (1959). Matrix Theory, Vol. 2. Chelsea Publishing Company, New York.

Gelfand, A. E., Hills, S. E., Racine-Poon, A., and Smith, A. F. M. (1990). Illustration of Bayesian Inference in Normal Data Models Using Gibbs Sampling. Journal of the American Statistical Association, 85:973-985.

Gelfand, A. E. and Smith, A. F. M. (1990). Sampling- Based Approaches to Calculating Marginal Densities. Journal of the American Statistical Association, 85:389-409.

Geman, S. and Geman, D. (1984). Stochastic Relaxation, Gibbs Distributions, and the Bayesian Restoration of Images. IEEE Transactions on Pattern Analysis and Machine Intelligence, 6:721-741. 
Ghosh, J. H. and Sen, P. K. (1985). On the Asymptotic Performance of the Log Likelihood Ratio Statistic for the Mixture Model and Related Results. In Cam, L. M. L. and Olshen, R. A., editors, Proceedings of the Berkeley Conference in Honor of Jery Neyman and Jack Kiefer, Vol. 2, 789-806. Wadsworth, Monterey.

Glosten, L. R., Jagannathan, R., and Runkle, D. E. (1993). On the Relation between the Expected Value and the Volatility of the Nominal Excess Return on Stocks. The Journal of Finance, 48:1779-1801.

Gouriéroux, C. (1997). ARCH Models and Fiancial Applications. Springer-Verlag, New York.

Gray, S. F. (1996). Modeling the Conditional Distribution of Interest Rates as a RegimeSwitching Process. Journal of Financial Economics, 42:27-62.

Hamilton, J. D. (1988). Rational-Expectations Econometric Analysis of Changes in Regime. An Investigation of the Term Structure of Interest Rates. Journal of Economic Dynamics and Control, 12:385-423.

Hamilton, J. D. (1989). A New Approach to the Economic Analysis of Nonstationary Time Series and the Business Cycle. Econometrica, 57:357-384.

Hamilton, J. D. and Susmel, R. (1994). Autoregressive Conditional Heteroskedasticity and Changes in Regime. Journal of Econometrics, 64:307-333.

Hansen, B. E. (1994). Autoregressive Conditional Density Estimation. International Economic Review, 35:705-730.

Hartigan, J. A. (1985). A Failure of Likelihood Asymptotics for Normal Mixtures. In Cam, L. M. L. and Olshen, R. A., editors, Proceedings of the Berkeley Conference in Honor of Jery Neyman and Jack Kiefer, Vol. 2, 807-810. Wadsworth, Monterey.

Harvey, C. R. and Siddique, A. (1999). Autoregressive Conditional Skewness. Journal of Financial and Quantitative Analysis, 34:465-487.

Henderson, H. V. and Searle, S. R. (1981). On Deriving the Inverse of a Sum of Matrices. SIAM Review, 23:53-60.

Jarque, C. M. and Bera, A. K. (1987). A Test for Normality of Observations and Regression Residuals. International Statistical Review, 55:163-172.

Kane, A. (1977). Skewness Preference and Portfolio Choice. Journal of Financial and Quantitative Analysis, 17:15-25.

Kass, R. E. and Raftery, A. E. (1995). Bayes Factors. Journal of the American Statistical Association, 90:773-795.

Kim, D. and Kon, S. J. (1994). Alternative Models for the Conditional Heteroscedasticity of Stock Returns. Journal of Business, 67:563-598.

Kon, S. J. (1984). Models of Stock Returns: A Comparison. The Journal of Finance, 39:147165.

Kuechler, U., Neumann, K., Soerensen, M., and Streller, A. (1999). Stock Returns and Hyperbolic Distributions. Mathematical and Computer Modelling, 29:1-15.

Leroux, B. G. (1992). Consistent Estimation of a Mixing Distribution. The Annals of Statistics, 20:1350-1360.

Lin, B.-H. and Yeh, S.-K. (2000). On the Distribution and Conditional Heteroskedasticity in Taiwan Stock Prices. Journal of Multinational Financial Management, 10:367-395.

Lütkepohl, H. (1991). Introduction to Multiple Time Series Analysis. Springer-Verlag, Heildelberg. 
Lux, T. (1997). Time Variation of Second Moments from a Noise Trader/Infection Model. Journal of Economic Dynamics and Control, 22:1-38.

Marron, J. S. and Wand, M. P. (1992). Exact Mean Integrated Squared Error. Annals of Statistics, 20:712-736.

McLachlan, G. J. and Basford, K. E. (1988). Mixture Models: Inference and Application to Clustering. Marcel Dekker, New York.

McLachlan, G. J. and Peel, D. (2000). Finite Mixture Models. John Wiley \& Sons, New York.

Mittnik, S. and Paolella, M. S. (2000). Conditional Density and Value-at-Risk Prediction of Asian Currency Exchange Rates. Journal of Forecasting, 19:313-333.

Mittnik, S., Paolella, M. S., and Rachev, S. T. (1998). Unconditional and Conditional Distributional Models for the Nikkei Index. Asia-Pacific Financial Markets, 5:99-128.

Mittnik, S., Paolella, M. S., and Rachev, S. T. (2000). Diagnosing and Treating the Fat Tails in Financial Returns Data. Journal of Empirical Finance, 7:389-416.

Mittnik, S. and Rachev, S. (1993). Modeling Asset Returns With Alternative Stable Models. Econometric Reviews, 12:261-330.

Neely, C. J. (1999). Target Zones and Conditional Volatility: The Role of Realignments. Journal of Empirical Finance, 6:177-192.

Nelson, D. B. (1991). Conditional Heteroskedasticity in Asset Returns: A New Approach. Econometrica, 59:347-370.

Nelson, D. B. (1995). Stationarity and Persistence in the GARCH(1,1) Model. In Engle, R. F., editor, ARCH. Selected Readings, 176-192. Oxford University Press, New York.

Nelson, D. B. and Cao, C. Q. (1992). Inequality Constraints in the Univariate GARCH Model. Journal of Business and Economics Statistics, 10:229-235.

Newcomb, S. (1980). A Generalized Theory of the Combination of Observations so as to Obtain the Best Result. In Stigler, M., editor, American Contributions to Mathematical Statistics in the Nineteenth Century, Vol. 2, 343-366. Arno Press, New York.

Pagan, A. R. and Schwert, G. W. (1990). Alternative Models for Conditional Stock Volatility. Journal of Econometrics, 45:267-290.

Palm, F. C. and Vlaar, P. J. G. (1997). Simple Diagnostic Procedures for Modeling Financial Time Series. Allgemeines Statistisches Archiv, 81:85-101.

Paolella, M. (1999). Tail Estimation and Conditional Modeling of Heteroscedastic Time-Series. Ph.D. Thesis, Institute of Statistics and Econometrics, University of Kiel. Pro Business, Berlin.

Paolella, M. S. (1997). Generalized GARCH Models with Nonnormal Distributions. Working Paper 111, Institute of Statistics and Econometrics, University of Kiel.

Peiró, A. (1999). Skewness in Financial Returns. Journal of Banking and Finance, 6:847-862.

Rockinger, M. and Jondeau, E. (2002). Entropy Densities with an Application to Autoregressive Conditional Skewness and Kurtosis. Journal of Econometrics, 106:119-142.

Roeder, K. and Wasserman, L. (1997). Practical Bayesian Density Estimation Using Mixtures of Normals. Journal of the American Statistical Association, 92:894-902.

Rosenblatt, M. (1952). Remarks on a Multivariate Transformation. Annals of Mathematical Statistics, 23:470-472. 
Rozelle, J. and Fielitz, B. (1980). Skewness in Common Stock Returns. Financial Review, $15: 1-23$.

Schwarz, G. (1978). Estimating the Dimension of a Model. Annals of Statistics, 6:461-464.

Sentana, E. (1995). Quadratic ARCH Models. Review of Economic Studies, 62:639-661.

Shleifer, A. and Summers, L. H. (1990). The Noise Trader Approach to Finance. Journal of Economic Perspectives, 4:19-33.

Simkowitz, M. and Beedles, W. (1980). Asymmetric Stable Distributed Security Returns. Journal of the American Statistical Association, 75:306-312.

St. Pierre, E. F. (1993). The Importance of Skewness and Kurtosis in the Time-Series of Security Returns. PhD thesis, Florida State University College of Business.

Stuart, A., Ord, J. K., and Arnold, S. (1999). Kendall's Advanced Theory of Statistics, Vol. 2A. Edward Arnold, London.

Teicher, H. (1963). Identifiability of Finite Mixtures. The Annals of Mathematical Statistics, 34:1265-1269.

Titterington, D. M., Smith, A. F., and Makov, U. E. (1985). Statistical Analysis of Finite Mixture Distributions. John Wiley \& Sons, Chichester.

Tucker, A. L. and Pond, L. (1988). The Probability Distribution of Foreign Exchange Price Changes: Tests of Candidate Processes. Review of Economics and Statistics, 70:638-647.

Vlaar, P. J. G. and Palm, F. C. (1993). The Message in Weekly Exchange Rates in the European Monetary System: Mean Reversion, Conditional Heteroscedasticity, and Jumps. Journal of Business and Economic Statistics, 11:351-360.

Wang, K.-L., Fawson, C., Barrett, C. B., and McDonald, J. B. (2001). A Flexible Parametric GARCH Model with an Application to Exchange Rates. Journal of Applied Econometrics, 16:521-536.

Wolfe, J. H. (1971). A Monte Carlo Study of Sampling Distribution of the Likelihood Ratio for Mixtures of Multinormal Distributions. Technical Bulletin 72-2, U.S. Naval Personnel and Training Research Laboratory, San Diego.

Wong, C. S. and Li, W. K. (2001). On a Mixture Autoregressive Conditional Heteroskedastic Model. Journal of the American Statistical Association, 96:982-995. 


\section{CFS Working Paper Series:}

\begin{tabular}{lll} 
No. & Author(s) & Title \\
\hline $2001 / 12$ & Daniel Gross & Trade Flows and the International Business Cycle
\end{tabular}

2002/01 Stefan Feinendegen

Daniel Schmidt

Mark Wahrenburg

2002/02 Issam Hallak

2002/03 Raimond Maurer

Christian Schlag

2002/04 Holger Claessen

Stefan Mittnik

2002/05 Bernd Kaltenhäuser

2002/06 Erik Theissen

2002/07 Werner Neus

Uwe Walz

2002/08 Andreas Bascha

Uwe Walz

2002/09 Marco Hoeberichts

2002/10 Markus Haas,

Stefan Mittnik

Marc S. Paolella
Die Vertragsbeziehung zwischen Investoren und Venture Capital-Fonds: Eine empirische Untersuchung des europäischen Venture Capital-Marktes

Price Discrimination on Syndicated Loans and the Number of Lenders: Empirical Evidence from the Sovereign Debt Syndication

Money-Back Guarantees in Individual Pension Accounts: Evidence from the German Pension Reform

Forecasting Stock Market Volatility and the Informational Efficiency of the DAX-index Options Market

Return and Volatility Spillovers to Industry Returns: Does EMU Play a Role?

Internalisierung und Marktqualität:

Was bringt Xetra Best?

Exit Timing of Venture Capitalists in the Course of an Initial Public Offering

Financing Practices in the German Venture

Capital Industry

An Empirical Assessment

The Credibility of Central Bank Announcements

Mixed Normal Conditional Heteroskedasticity

Copies of working papers are available at the Center for Financial Studies or can be downloaded (http://www.ifk-cfs.de). 